\title{
Mirror production for the Cherenkov telescopes of the ASTRI mini-array and the MST project for the Cherenkov Telescope Array
}

\author{
Nicola La Palombara $\odot,{ }^{\text {a,* }}$ Giorgia Sironi, ${ }^{\text {b }}$ Enrico Giro $\odot,{ }^{\mathrm{c}}$ \\ Salvatore Scuderi $\odot,{ }^{\mathrm{a}}$ Rodolfo Canestrari $\odot{ }^{\mathrm{d}}$, Simone Iovenitti $\odot,{ }^{\mathrm{b}, \mathrm{e}}$ \\ Markus Garczarczyk $\odot,{ }^{\mathrm{f}}$ Maria Krause, ${ }^{\mathrm{f}}$ Sebastian Diebold, ${ }^{\mathrm{g}}$ \\ Rachele Millul $\odot$, ${ }^{\text {F }}$ Fabio Marioni, ${ }^{\text {h }}$ Nadia Missaglia,, Matteo Redaelli, \\ Giuseppe Valsecchi, ${ }^{\text {h }}$ Fabio Zocchi, ${ }^{\text {h }}$ Adelfio Zanoni, ${ }^{i}$ and \\ Giovanni Pareschi $\odot^{b}$ \\ ${ }^{a}$ INAF-IASF Milano, Milano, Italy \\ ${ }^{\mathrm{b}}$ INAF-Osservatorio Astronomico di Brera, Merate, Italy \\ ${ }^{\mathrm{c}}$ INAF-Osservatorio Astronomico di Padova, Padova, Italy \\ ${ }^{\mathrm{d}}$ INAF-IASF Palermo, Palermo, Italy \\ ${ }^{\mathrm{e} U n i v e r s i t a ̀ ~ d e g l i ~ S t u d i ~ d i ~ M i l a n o, ~ D i p a r t i m e n t o ~ d i ~ F i s i c a, ~ M i l a n o, ~ I t a l y ~}$ \\ ${ }^{\mathrm{f}}$ Deutsches Elektronen-Synchrotron, Zeuthen, Germany \\ ${ }^{\mathrm{g}}$ Institut für Astronomie und Astrophysik Tübingen, Tübingen, Germany \\ ${ }^{\mathrm{h}}$ Media Lario s.r.l., Bosisio Parini, Italy \\ iZAOT s.r.l., Vittuone, Italy
}

\begin{abstract}
The Cherenkov Telescope Array (CTA) is the next ground-based gamma-ray observatory in the $\mathrm{TeV} \gamma$-ray spectral region operating with the Imaging Atmospheric Cherenkov Technique. It is based on almost 70 telescopes of different class diametersLarge-Sized Telescope, Medium-Sized Telescope (MST), and Small-Sized Telescope (SST) of 23, 12, and $4 \mathrm{~m}$, respectively - to be installed in two sites in the two hemispheres (at La Palma, Canary Islands, and near Paranal, Chile). Several thousands of reflecting mirror tiles larger than $1 \mathrm{~m}^{2}$ will be produced for realizing the segmented primary mirrors of a so large number of telescopes. Almost in parallel, the ASTRI mini-array (MA) is being implemented in Tenerife (Canary Islands), composed of nine 4-m diameter dual-mirror Cherenkov telescopes (very similar to the SSTs). We completed the mirror production for all nine telescopes of the ASTRI MA and two MSTs (400 segments in total) using the cold glass slumping replication technology. The results related to the quality achieved with a large-scale production are presented, also discussing the adopted testing methods and approaches. They will be very useful for the adoption and optimization of the quality assurance process for the huge production (almost $3000 \mathrm{~m}^{2}$ of reflecting surface) of the MST and SST CTA telescopes. @ The Authors. Published by SPIE under a Creative Commons Attribution 4.0 International License. Distribution or reproduction of this work in whole or in part requires full attribution of the original publication, including its DOI. [DOI: 10.1117/1.JATIS.8.1.014005]
\end{abstract}

Keywords: ASTRI; Cherenkov Telescope Array; Cherenkov; $\boldsymbol{\gamma}$-ray; cold-slumping technology; hot-slumping technology; quality assurance.

Paper 21052 received May 4, 2021; accepted for publication Jan. 14, 2022; published online Feb. 3, 2022.

\section{Introduction}

Imaging Atmospheric Cherenkov Telescopes (IACTs) are ground-based telescopes designed to observe $\gamma$-ray celestial sources at very-high energies (VHE) in the $\mathrm{TeV}$ energy range. ${ }^{1}$ After the first detection of a VHE source, obtained in 1989 with the Whipple telescope, ${ }^{2}$

*Address all correspondence to Nicola La Palombara, nicola.lapalombara@inaf.it

${ }^{\dagger}$ For the ASTRI (http://www.astri.inaf.it/) and the CTA (https://www.cta-observatory.org/) projects. 
several other sources have been found over the last three decades, thanks to the H.E.S.S., ${ }^{3}$ MAGIC, ${ }^{4}$ and VERITAS ${ }^{5}$ telescopes. Currently, more than 200 VHE sources are known: ${ }^{6}$ some of them have been identified with different classes of celestial objects, but a significant fraction of VHE sources are still unidentified, ${ }^{7}$ in addition, due to the limited sensitivity of the current generation of instruments, in most cases, the origin of the observed VHE emission is not fully understood. Therefore, new facilities with better performance are necessary.

ASTRI is a project led by the Italian National Institute for Astrophysics (INAF) that aims to realize a series of dual-mirror IACTs. They are characterized by innovative technological solutions, such as the Schwarzschild-Couder optical configuration, ${ }^{8}$ a modular, light, and compact focal-plane camera consisting of an array of multipixel silicon photomultiplier sensors, and efficient and fast front-end electronics, specifically designed for ASTRI. The feasibility of this innovative design has been successfully demonstrated with the ASTRI-Horn prototype. This telescope, installed in 2014 at the Serra La Nave site on Mount Etna (Catania, Italy), is the first Schwarzschild-Couder telescope to be built and tested: ${ }^{9}$ It observed its first light in May $2017^{10}$ and detected the Crab nebula in December 2018. ${ }^{11}$

On behalf of INAF, the ASTRI collaboration is developing a mini-array (ASTRI MA) composed of nine dual-mirror IACTs, based on the design of the ASTRI-Horn prototype. ${ }^{12,13}$ They will be installed at the Teide Astronomical Observatory, operated by the Instituto de Astrofisica de Canarias (IAC), on Mount Teide, in the Canary island of Tenerife. The ASTRI MA will be operated by INAF based on a host agreement with IAC. ${ }^{14,15}$

In addition, the ASTRI Collaboration is participating to the preparatory effort for the realization of the Cherenkov Telescope Array (CTA). It will be located in two different sites, one in the northern and one in the southern hemisphere, and will be made up of three different types of IACTs. ${ }^{16}$

- The Large-Sized Telescopes (LSTs), with a 23-m mirror diameter, will cover the lowenergy range between $20 \mathrm{GeV}$ and $3 \mathrm{TeV}$.

- The Medium-Sized Telescopes (MSTs), with a 12-m mirror diameter, will observe the core energy range between $80 \mathrm{GeV}$ and $50 \mathrm{TeV}$.

- The Small-Sized Telescopes (SSTs), with a 4-m primary mirror diameter, will be devoted to the high-energy range from $\sim 1 \mathrm{TeV}$ up to more than $300 \mathrm{TeV}$.

With respect to the initial design configuration that was based on almost 100 telescopes, ${ }^{17}$ the CTAO arrays have recently been descoped: the baseline configuration, dubbed as "alpha configuration," will consist of a Northern Array with 4 LSTs and 9 MSTs and a Southern Array with 14 MSTs and 37 SSTs.

The improvement in the on-axis differential sensitivity of the two CTAO Arrays with respect to the currently existing instruments of the same kind (mainly MAGIC, H.E.S.S., and VERITAS) is a factor of 5 to 10 depending on the energy range. (Public CTAO webpage reporting a full set of performance plots for the alpha configuration can be found in Refs. 18 and 19.) Particularly, relevant is the CTAO improvement in terms of off-axis sensitivity. Between 3 deg and 4 deg away from the pointing direction the differential sensitivity worsens just by less than a factor 2 at all energies with respect to the on-axis sensitivities. CTAO therefore has a $\gamma$-ray field of view (FoV) of 7-deg in diameter at $1 \mathrm{TeV}$ (even more at higher energies).

It should be noted that the performance of CTAO would also be improved, after the implementation of this alpha configuration, with the installation of additional telescopes, in particular for the southern site, where there is a large area that could be populated with additional telescopes. In this respect, it should be noted that another kind of medium size telescopes, based on the dual-mirror Schwarzschild-Couder aplanatic telescopes (9- $\mathrm{m}$ in diameter) and named prototype Schwarzschild-Couder Telescope (pSCT), would be possibly implemented, similar to the prototype already developed and operated in Arizona (USA). ${ }^{20,21}$

In the context of CTA, INAF is leading the international collaboration ${ }^{22,23}$ that will provide, in terms of in-kind contribution to CTAO, the SSTs. The design will be based on that of the ASTRI-Horn prototype and of the ASTRI MA telescopes. The mirrors will be provided by INAF and they will be of the same type of the ASTRI MA. 
The MSTs are based on a single-mirror Davies-Cotton optical design. ${ }^{24}$ Each of the $12-\mathrm{m}$ primary mirrors will be made assembling together 86 identical hexagonal segments with spherical profile and a maximum size of $120 \mathrm{~cm}$. INAF, in the context of the ASTRI collaboration, is also responsible for the procurement of all the reflecting segments for the 9 MSTs that will be implemented at the northern site ( 800 segments to be produced, including the spares). The sandwiched mirror segments are produced using the so-called glass cold-slumping replication technology, initially set-up by INAF-Osservatorio Astronomico di Brera (OAB) and Media Lario (ML) company (Bosisio Parini, Italy) for the MAGIC II telescope ${ }^{25}$ and then specifically developed to fit the requirements and specifications of the MSTs. ${ }^{26}$ In this respect, ASTRI is cooperating with the Deutsches Elektronen-Synchrotron (DESY, Germany), responsible for the MST structures.

Also, the reflecting segments of the primary mirrors for the ASTRI MA are produced using the same technology $\mathrm{y}^{27}$ and they will be used for the production of segments for the primary mirrors of the 37 (extendable to 40) SSTs to be installed at the southern site (with about other 800 segments to be produced, including the spares). Each primary is based on 18 hexagonally shaped segments, with a maximum size of $85 \mathrm{~cm}$.

At the end of 2019, we completed the mirror production for both the nine telescopes of the ASTRI MA (200 reflecting segments of three different curvature radii and profile, corresponding to the three coronas of segments to form a primary) and two MSTs (with 200 identical reflecting segments with spherical shape produced). In this effort, the ML Company represents the industrial partner, while ZAOT (Vittuone, Italy) is involved for the application of the reflective coating on the substrates. In this context, we have performed independent verifications of the optical performance of representative mirror samples.

In this paper, the aspects related to the large-scale production of these segments are presented, with a particular regard to the qualification activities that have been performed in order to assess and consolidate the production process. In this respect, the criteria adopted for the quality assurance, in order to monitor and verify the production reliability, are discussed in terms of performance and compliance with respect to the input requirements. Different methods and techniques have been used to perform the verifications. Finally, we present the performance of the produced segments and discuss their compliance and production yield with respect to the input requirements. The results of the present work will be very useful for the adoption and optimization of the quality assurance process for the huge production of the MST north and SST CTA telescopes, for which thousands of reflecting segments have to be manufactured and qualified.

\section{Optical Design}

\subsection{Optical Design of the Telescopes for ASTRI MA}

The dual-mirror telescopes of the ASTRI MA are based on the polynomial SchwarzschildCouder optical design, an aplanatic layout that, thanks to the combination of the two mirrors, is characterized by a wide field of view, a low vignetting and isochrony. The plate scale of the ASTRI telescopes is $37.6 \mathrm{~mm} / \mathrm{deg}$.

The primary mirror (ASTRI M1) has a radial symmetry and its optical profile is described by an aspherical polynomial function (of order 10), where the radius of the spherical component is $8.223 \mathrm{~m}$. It has a diameter of $4.3 \mathrm{~m}$ and is segmented into 18 hexagonal segments; the face-to-face dimension of each segment is $85 \mathrm{~cm}$. They are distributed in three concentric coronae (COR1, COR2, and COR3) of six identical segments each, where each corona has a different radial distance from the telescope axis. In fact, the best-fit radius of curvature (RoC) of the segments is 8.6, 9.8, and $11.7 \mathrm{~m}$, respectively, for the inner, medium, and outer corona. $^{28}$

The secondary mirror (ASTRI M2) is a monolithic element and has a diameter of $1.8 \mathrm{~m}$. It has an aspherical shape, with a radius of $2.18 \mathrm{~m}$ for the spherical component, and is installed at a distance of $3.108 \mathrm{~m}$ from ASTRI M1. This implies an equivalent focal length for the whole telescope of $2.15 \mathrm{~m}^{22}$ 


\subsection{Optical Design of the Single-Mirror MST}

The medium-sized telescope has a single-mirror configuration with a modified Davies-Cotton optical design, ${ }^{29}$ where the optical parameters are optimized to reduce the time spread at the Cherenkov camera focal plane from light reflected at different mirror positions. The mirror is segmented into 86 hexagonal segments, for a diameter of $12 \mathrm{~m}$. All segments are identical: they have a spherical profile, with an RoC of $32.14 \mathrm{~m}$, and the face-to-face size is $1.2 \mathrm{~m}$. The telescope focal length is $16 \mathrm{~m}$, and the mirror segments are aligned to reflect rays parallel to the optical axis into the focal point. They are mounted on a spherical dish with a radius of $19.2 \mathrm{~m}$. The plate scale of the MSTs is $280 \mathrm{~mm} / \mathrm{deg}$.

\section{Mirror Design and Production}

In the framework of CTA, several different methods have been considered for the mirror realization, which are based on different technologies: ${ }^{30-33}$ they include sandwich concepts with cold-slumped surfaces made of thin float glass and different core materials (such as aluminum honeycomb, glass foams, or aluminum foams), constructions based on carbon fiber/epoxy or glass fiber substrates, as well as sandwich structures made entirely of aluminum. In addition, lightweight polished aluminum mirrors with $\mathrm{Ni} / \mathrm{P}$ alloy ("electroless nickel") cladding as the reflecting surface were proposed for the Gamma-ray Cherenkov Telescope, the other dualmirror telescope with a Schwarzschild-Couder optical design ${ }^{34}$ that was proposed for the SSTs before the selection of the ASTRI design as the baseline. In this respect, the electroless nickel $^{35}$ [composed of nickel $(\mathrm{Ni})$ and $9 \%$ to $12 \%$ of phosphorus $(\mathrm{P})$ ] is often added as a cladding layer of a few tens of micrometers onto the aluminum-based surface because of its hard and-at the same time-amorphous microstructure, which makes easier the super-polishing process of the optical surface. This allows us to achieve very low microroughness levels on metallic surfaces [up to a fraction of nanometer root-mean squared (RMS), see Refs. 36 and 37].

Both the ASTRI M1 segments and the MST segments are realized using an improved version of glass cold-slumping technology (in this context, however, it should be noted that part of the mirrors for MST are being realized also with alternative technologies) that was developed in synergy between OAB and ML. This approach has been already successfully used for the two telescopes of the MAGIC experiment array operated at the Observatorio di Roque de Los Muchacos astronomical site (La Palma, Canary Islands). ${ }^{25,38,39}$ The main steps of the mirror production are shown in Fig. 1. The cold slumping is used to bend a thin glass foil onto a mould

(a)

Preparation of the integration mold

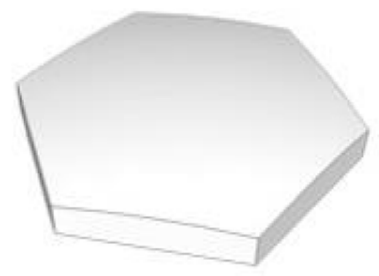

(d)

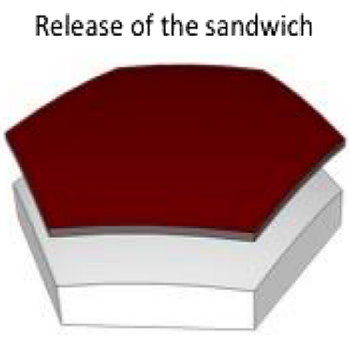

(b)

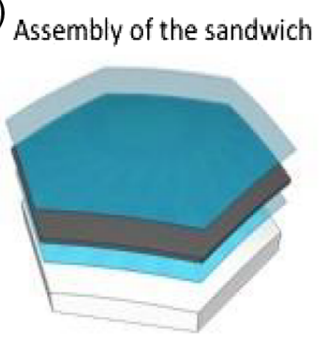

(e)

Coating of the sandwich

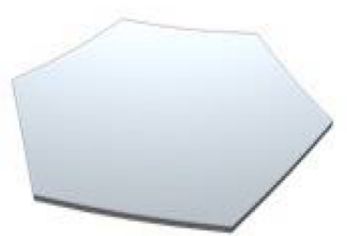

(c)

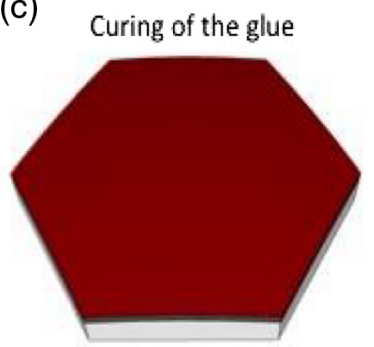

(f)

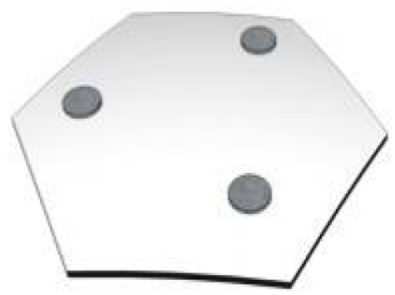

Fig. 1 Conceptual description of the (a)-(f) main steps of the cold-slumping technology. 
(a)

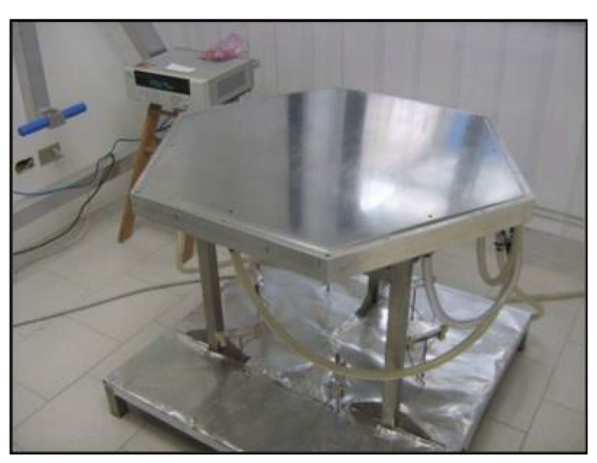

(c)

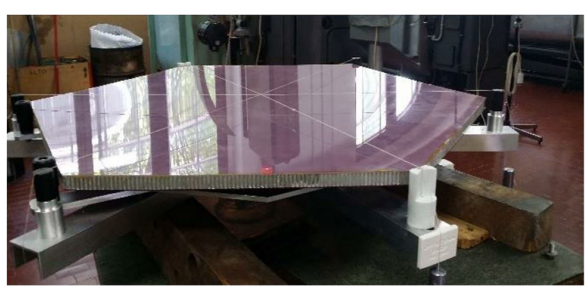

(b)

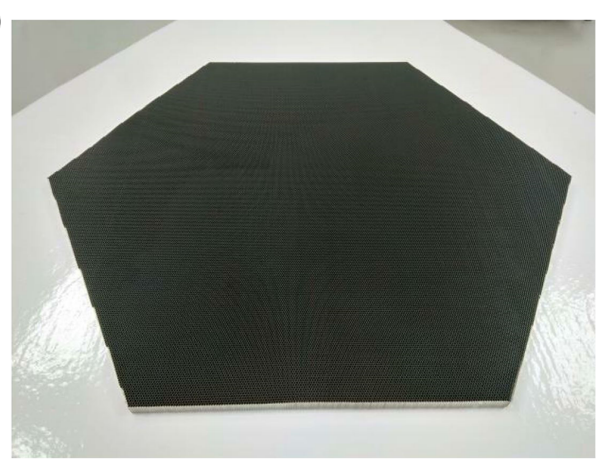

(d)

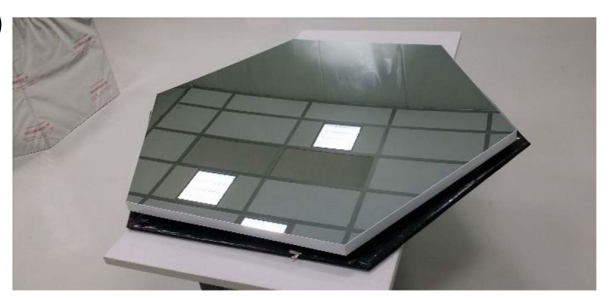

Fig. 2 Pictures of the main steps of the mirror production with the cold-slumping method: (a) bending of a thin glass foil onto the reference mould; (b) honeycomb layer of aluminum to be glued onto the glass foil; (c) sandwich structure of two glass foils with an Al honeycomb core; and (d) final mirror, with the reflective coating applied onto the first glass foil.

with the desired profile [Fig. 2(a)]. Then a honeycomb layer of aluminum [Fig. 2(b)] is glued onto the glass foil and, afterward, a second glass foil is glued onto the honeycomb layer. In this way, two glass foils are assembled in a light but stiff sandwich structure with an Al honeycomb core [Fig. 2(c)]. After the curing of the glue, the sandwich is released and a highly reflective coating is applied to the outer surface of the inner glass foil [Fig. 2(d)]. All the segments of the primary mirrors for the ASTRI MA and MSTs are realized using this technology, adapted to the two different cases. ${ }^{26}$

In the case of the ASTRI M1 segments, the thickness of the glass foils is $1.6 \mathrm{~mm}$, while that of the honeycomb layer is $20 \mathrm{~mm}$; for the MST segments, the corresponding values are 2.1 and $30 \mathrm{~mm}$, respectively. The reflective surface is a multilayer coating composed of $\mathrm{Al}+\mathrm{SiO}_{2}+$ $\mathrm{ZrO}_{2}+\mathrm{SiO}_{2}$. The advantage of this technology is that it is based on a cost-saving replica process, which is suitable for CTA due to the multiplicity of the telescope mirrors. The chemical composition of the reflective layer guarantees a reflectivity profile higher than $85 \%$ in the wavelength range between 300 and $550 \mathrm{~nm}$. Moreover, the evaporation process used to apply the reflective coating onto the mirror ensures a high-reflectivity uniformity.

The segments obtained are lightweight (about $10 \mathrm{~kg} / \mathrm{m}^{2}$, excluding the three supporting pads, made in stainless steel, that increase the mass of less than two additional kilograms) and with high surface accuracy, which is measured with two different parameters, the microroughness and the residual shape error, both contributing in worsening the point spread function (PSF). Both parameters are expressed as the RMS deviation of the reflecting surface from its ideal shape in the normal direction: but in the case of the microroughness, the deviation is measured on microscopic spatial scales (with spatial wavelengths from a few micrometers up to $1 \mathrm{~mm}$ ) at high spatial frequencies ${ }^{40}$ while in the case of the shape error the deviation is measured at longer spatial scales. Surface microroughness affects the optical performance by degrading the PSF and reducing the image contrast, ${ }^{41}$ while the residual shape error causes deviation from the ideal focusing directions due to the geometrical imperfections. In accordance to the study performed by Taybaly et al., ${ }^{40}$ the microroughness shall be $<7.0 \mathrm{~nm} \mathrm{RMS}$, as measured in the 7 - to $500-\mu \mathrm{m}$ spatial wavelength range, in order to avoid spread of scattered photons $>15 \%$ beyond a diameter of $0.12 \mathrm{deg}$ of the focal spot.

The accuracy of the mirror shape much depends on the accuracy of the reference moulds used for replication process, which have a residual shape error $<5 \mu \mathrm{m}$ RMS compared with the 
expected shape. Since the replica process introduces a deviation lower than a factor 5 from the mould shape, the produced mirrors are characterized by a residual shape error $<25 \mu \mathrm{m}$ RMS that is well within usual requirements for the CTA telescopes.

Regarding the ASTRI M2 mirror, the system is based on a monolithic glass substrate with a thickness of $19 \mathrm{~mm}$. This solution allows us to simplify the telescope design but requires a thick glass substrate, which cannot be curved to the required RoC with the cold-slumping technology used for the primary segments. Therefore, the ASTRI M2 mirror is bent to the correct shape using the hot-slumping technology. The reflective surface is obtained with the application of the same multilayer coating used for the ASTRI M1 and MST segments. Since the ASTRI M2 mirrors are produced with a completely different technology, the relevant production and qualification activities will not be described in this paper.

As mentioned in Sec. 1, the CTA southern array could be possibly extended after the implementation of the baseline configuration. In this context, additional medium size $-9-\mathrm{m}$ diameter-aplanatic telescopes may be added with dual-mirror Schwarzschild-Couder configuration. ${ }^{8}$ This design is capable to minimize the spherical and comatic aberrations across a large field of view together with a reduction of the astigmatism by adopting a bended focal plane detection surface. An end-to-end prototype (pSCT) has been successfully developed in US, Arizona, ${ }^{20}$ at the site at the Fred Lawrence Whipple Observatory. The telescope optical system of pSCT consists of a 9.7- $\mathrm{m}$ diameter primary mirror and a 5.4-m secondary. The primary is based on 48 individual mirror asymmetric petals: an inner and an outer rings of 16 and 32 asymmetric petals, respectively (with an average area of $1.2 \mathrm{~m}^{2}$ ). The secondary reflector is also segmented, with 8 petals forming an inner ring and 16 petals forming the outer ring (with an average area of $0.94 \mathrm{~m}^{2}$ ). It should be noted that the reflecting panels have been realized by ML, supported also by INAF, using a glass replication technology almost identical to the production method adopted for the mirrors of ASTRI MA e MST. However, in the case of the innermost panels of the secondary mirror, due to the large sag of the segments, thin thermally preshaped glass foils have been used ${ }^{42}$ instead of adopting a pure cold replication method. The use of preshaped glass foils via hot slumping for making the sandwiched panels presented a few challenging aspects, in particular related to the formation of microcracks on the surface of the glass that made the material less resistant to the stresses generated during the replication process. The pSCT telescope has been successfully tested and calibrated. Its functionality has been demonstrated with the characterization of the imaging capability ${ }^{43}$ and the detection of the Crab nebula in gamma rays. ${ }^{44}$

A couple of other cold replication approaches, and alternative to the one proposed by INAF and ML, have been proposed for making the reflecting segments of the MSTs. In particular, the solution based on open-structure composite segmented mirrors has been investigated by the Institute of Nuclear Physics of the Polish Academy of Sciences. ${ }^{45}$ Another similar method, but based on a close sealed structure, is the one investigated at IRFU-Saclay (France), with the panels made of layers of different materials. ${ }^{46}$ At the time being, the two groups are engaged in an effort aiming at merging together the competences developed by the two groups, in order to deliver together all the reflecting panels for the 14 MSTs of the CTAO Southern Array.

In the case of the CTA LSTs, the mirrors are manufactured using the cold slumping technique and have a sandwich structure consisting of an aluminum honeycomb between two glass sheets, with a technology pretty similar to the one developed by INAF and ML. ${ }^{47,48}$ The parabolic primary mirror of CTA-LST is $23-\mathrm{m}$ diameter and its focal length is $28 \mathrm{~m}$. The primary mirror consists of 198 segmented mirrors. Each mirror has a hexagonal shape of 1.51-m side-by-side size. All the mirrors for the first LST have been already produced and successfully installed.

It should be noted that by Will et al., ${ }^{49}$ in collaboration with ML, are testing a new kind of back-coated glass mirrors for the MAGIC telescopes. This technology represents an evolution of the INAF glass replication approach, but in this case a protective layer of an ultra-thin glass sheet which is back coated with aluminum is used instead of the $\mathrm{SiO}_{2}$ thin coating deposited under vacuum onto the aluminum reflecting layer. Finally, another promising replication process under investigation by INAF and ML foresees a new fabrication scheme, similar to the cold glass replication, but where the reflecting slab is a low-cost laminate precoated reflecting aluminum strips instead of glass sheets [G. Pareschi, et al., Patent Application number: 102021000019658 submitted on 23 July 2021 to the Ministero dello Sviluppo Economico of the Italian Government

J. Astron. Telesc. Instrum. Syst. $\quad$ 014005-6 Jan-Mar 2022 • Vol. 8(1) 
with the title "Metodo per la costruzione di un elemento ottico per un telescopio ed elemento ottico ottenuto con tale metodo" (in English: Method for the construction of an optical element for a telescope and optical element obtained with such a method) $].{ }^{50}$

\section{Applicable Requirements}

For the development of the manufacturing process for the mirror segments, the CTA requirements have been assumed, not only for the case of the MST tiles but also for the one of the ASTRI MA (which are the same of the SSTs). These reference parameters regard not only the optical performance of each item but also its reliability, taking also into account the environmental conditions of the observing sites (Table 1) and the durability prescriptions.

From the performance point of view, the initial reflectivity of all facets, at any wavelength in the reference range 300 to $550 \mathrm{~nm}$, must be $>85 \%$. This requirement should be fulfilled also for the secondary mirrors of the ASTRI MA (and therefore CTA/SST) telescopes. In this respect, the rays, after the first reflection by the primary, impinge the secondary mirrors with angles between $10 \mathrm{deg}$ and $50 \mathrm{deg}$ with respect to the optical axis. On the other hand, the theoretical reflectivity of aluminum-coated mirrors with $\mathrm{SiO}_{2}$ protection (utilized for the monolithic secondary mirrors and deposited via evaporation ${ }^{51}$ ) remains very high within this entire angular range; experimental verifications were also carried out on witness samples.

In addition, there is a requirement on the angular size of the telescope PSF. To this aim, the reference parameter is $\theta_{80}$, which is the angular diameter of the circle that includes the $80 \%$ of the

Table 1 Applicable CTA environmental requirements.

\begin{tabular}{|c|c|}
\hline Requirement description & Value \\
\hline Scientific observations shall be possible when air temperature is: & $-15^{\circ} \mathrm{C}<T<+25^{\circ} \mathrm{C}$ \\
\hline Telescopes shall suffer no damage when air temperature is: & $-20^{\circ} \mathrm{C}<T<+40^{\circ} \mathrm{C}$ \\
\hline Telescopes shall suffer no damage when air temperature gradient at night time is: & $<7.5^{\circ} \mathrm{C} / \mathrm{h}$ \\
\hline Telescopes shall suffer no damage when air temperature variations within $24 \mathrm{~h}$ are: & $\pm 30^{\circ} \mathrm{C}$ \\
\hline Telescopes shall withstand continuous air temperature gradients of at least: & $0.72^{\circ} \mathrm{C} / \mathrm{h}$ for $25 \mathrm{~min}$ \\
\hline Scientific observations shall be possible when humidity is: & $2 \%$ to $90 \%$ \\
\hline Telescopes shall suffer no damage when humidity is: & $2 \%$ to $100 \%$ \\
\hline Telescopes shall suffer no damage when rain in $24 \mathrm{~h}$ is: & $<200 \mathrm{~mm}$ \\
\hline Telescopes shall suffer no damage when rain in $1 \mathrm{~h}$ is: & $<70 \mathrm{~mm}$ \\
\hline $\begin{array}{l}\text { Telescopes shall suffer no damage for precipitation in the form of rain, snow, } \\
\text { and hail when wind speed is: }\end{array}$ & $<90 \mathrm{~km} / \mathrm{h}$ \\
\hline Telescopes shall suffer no damage during slewing to safe position when rain is: & $<0.5 \mathrm{~mm} / \mathrm{min}$ \\
\hline Telescopes shall suffer no damage when snow accumulation on ground is: & $<50 \mathrm{~cm}$ \\
\hline Telescopes shall suffer no damage by the impact of hailstones with diameter: & $<20 \mathrm{~mm}$ \\
\hline Telescopes shall suffer no damage when the ice thickness (on all surfaces) is: & $<20 \mathrm{~mm}$ \\
\hline Scientific observations shall be possible when wind speed is: & $<36 \mathrm{~km} / \mathrm{h}$ \\
\hline $\begin{array}{l}\text { Telescopes shall suffer no damage during slewing to safe position } \\
\text { when wind speed is: }\end{array}$ & $<50 \mathrm{~km} / \mathrm{h}$ \\
\hline Telescopes shall suffer no damage when the 10-min average wind speed is: & $<120 \mathrm{~km} / \mathrm{h}$ \\
\hline Telescopes shall suffer no damage when the 1-s average wind gusts are: & $<200 \mathrm{~km} / \mathrm{h}$ \\
\hline
\end{tabular}


focused photons. For SST (and therefore also ASTRI MA) and MSTs, the CTA requirement is $\theta_{80}<0.25 \mathrm{deg}$ and $\theta_{80}<0.18 \mathrm{deg}$, respectively.

It should be noted that the PSF requirement for the MST is strictly referred just to the on-axis direction, in order to allow the quality assurance verification of each spherical tile. Indeed, while the SSTs (and ASTRI MA) are aplanatic within their FoV, one should be reminded that the MST optical system is instead affected by an inherent off-axis degradation of the PSF, as they are based on the single-mirror Davies-Cotton design. ${ }^{33}$ A worsening of the angular resolution is then associated with the increase of the off-set angles and the PSF also becomes asymmetric in the two directions, with the spot size, which is always larger in the radial direction. This is mostly due to the non-Gaussian tails of the PSF and the effect tends to be more enhanced with the increase of the focal length/diameter ratio. At the border of the FoV of the MSTs $( \pm 3.7 \mathrm{deg})$, the maximum degradation due to the design will be about 0.10 deg, i.e., in any case lower than the angular resolution requirement. Therefore, also for off-axis rays, the PSF of the MST is expected to be dominated by the fabrication and alignment errors.

The main environmental requirements are reported in Table 1; furthermore, there are also requirements on the resistance to aggressive atmosphere, dust and sand blasting, and lightning. Finally, the provided mirrors and segments must have a lifetime $>15$ year. Of course, this last parameter could not be directly proven within the activities and tests performed and reported in this work. However, the experience has been coming from the glass sandwiched mirrors on the MAGIC II telescope since 2007, that, up to now, have shown a very small degradation, and the aging tests performed so far indirectly indicate that this requirement can be met.

In view of the mirror production, all the CTA environmental requirements reported in Table 1 were considered as applicable requirements for ML, the industrial supplier of MST, and ASTRI M1 segments. We only reinforced the air temperature range where telescopes shall suffer no damage $\left(-20^{\circ} \mathrm{C}<T<+70^{\circ} \mathrm{C}\right)$, to take into account also the transport and storage conditions before the segment integration on the telescope. Moreover, we required that the mirror segments are waterproof and that the reflective coating can survive to a tape adhesion test with a pulling force up to $16 \mathrm{~N}$. Finally, from the reliability point of view, we required that the lifetime of the reflective coating is $>6$ year, with an end-of-life reflectivity $>65 \%$ in the reference wavelength range. A number of aging tests on the coating were performed, ${ }^{27}$ while one should note that the reflectivity loss of the mirrors mounted on MAGIC II at the Observatorio del Roque de los Muchachos (i.e., the same astronomical site in La Palma, Canary islands, where the northern array of CTA will be operated) was $<2 \%$ after more than 5 years. ${ }^{52}$ Recent tests performed on an MST mirror that was mounted on a truss in Paranal (Chile) at the site where the CTA southern array will be installed and did not show any degradation after 1 year of exposition to the local environment. ${ }^{53}$

From the performance point of view, we required an initial reflectivity value $>85 \%$ in the wavelength range 300 to $550 \mathrm{~nm}$. In addition, we required a maximum value $<8 \%$ (over the entire mirror surface) for the reflectivity nonuniformity, which is defined as the maximum difference between the average surface reflectivity measured at different positions over the mirror. Concerning the microroughness, the criterion adopted for the production was to maintain the already excellent RMS level $<2 \mathrm{~nm}$, i.e., a value much lower than the maximum acceptable value of $7 \mathrm{~nm}$ RMS estimated by Tayabaly et al. ${ }^{40,54}$ in order to not exceed the MST $\theta_{80}$ requirement because of scattering.

We required that the microroughness be $<2 \mathrm{~nm}$ RMS, thus constraining the dispersion of peaks and valleys of the real surface profile. For the MST segments, we required a $D_{80}$ (the linear dimension of $\left.\theta_{80}\right)<12 \mathrm{~mm}$ at the nominal focal (NF) length for the on-axis single mirror, which is half of the RoC (due to the spherical shape of the mirror). For a focal length of $16.07 \mathrm{~m}$, this corresponds to about $0.045 \mathrm{deg}$, which is well within the CTA requirement. Regarding the radius of curvature, we considered as a guideline that $\mathrm{RoC}=32.14 \pm 0.04 \mathrm{~m}$. For the ASTRI M1 segments, there is no requirement on the RoC, since they are aspheric. In their case, we introduced two requirements, one on the radius of the spherical component (between 8198 and $8298 \mathrm{~mm})$ and one on the residual shape error $(<20 \mu \mathrm{m}$ RMS for corona 1 and $<25 \mu \mathrm{m}$ RMS for coronae 2 and 3). Finally, for both the MST and ASTRI M1 segments the tolerance on the hexagon side-to-side size is $\pm 2 \mathrm{~mm}$. 


\section{Qualification of the Production Process}

Before starting with the mass production of the mirror segments, it was necessary to validate the production process and to perform qualification tests on a set of preliminary segments. To this end, the industrial activity started in February 2017 with the development phase. During this phase, the moulds used to produce the different types of segments were qualified and the parameters of the glass cold-shaping process were fine-tuned, in order to satisfy the performance requirements of the mirrors. Several segment prototypes were then produced and tested along this period, in order to ascertain that the production process was stable and repeatable. Afterward, one qualification segment of each type went through a complete set of performance measurements and environmental and reliability tests. During the qualification process, the following environmental tests were performed on each qualification segment:

(1) five thermal cycles composed of the following steps:

- $\quad$ from $20^{\circ} \mathrm{C}$ to $-20^{\circ} \mathrm{C}$;

- $\quad$ plateau of $12 \mathrm{~h}$;

- $\quad$ from $-20^{\circ} \mathrm{C}$ to $70^{\circ} \mathrm{C}$;

- $\quad$ plateau of $4 \mathrm{~h}$;

- $\quad$ cool down to $20^{\circ} \mathrm{C}$.

For all steps, the temperature gradient is $0.125^{\circ} \mathrm{C} / \mathrm{min}$ :

(2) dry heat test [according to ISO 9022-2:2002(E)];

(3) dump heat test [according to ISO 9022-2:2002(E)].

Before and after each of the aforementioned tests, the tested segment was characterized in terms of integrity, reflectivity, and residual shape error map. This was computed as difference between the measured shape, verified with a POLI TCX coordinate measurement machine (CMM) on a 10-mm square grid and the designed one. In all cases, after the test: (1) the visual inspection revealed no degradation in terms of panel integrity and quality; (2) the measured reflectivity was fully compatible with the original one; and (3) the comparison of the residual maps revealed no significant variation of the surface shape error. Moreover, the immersion in a water tank was executed, in order to verify the water tightness.

Regarding the reflecting coating, the reflectivity curve on the wavelength range 300 to $550 \mathrm{~nm}$ was measured on 13 equidistant points along three diagonals (Fig. 3), in order to sample peripheral, intermediate, and central areas of the mirror. The adhesion of the mirror coating was verified through a tape removal test. Both the reflectivity measurements and the tape test were repeated after the execution of thermal cycles, dry- and damp-heat tests, and solar radiation tests.

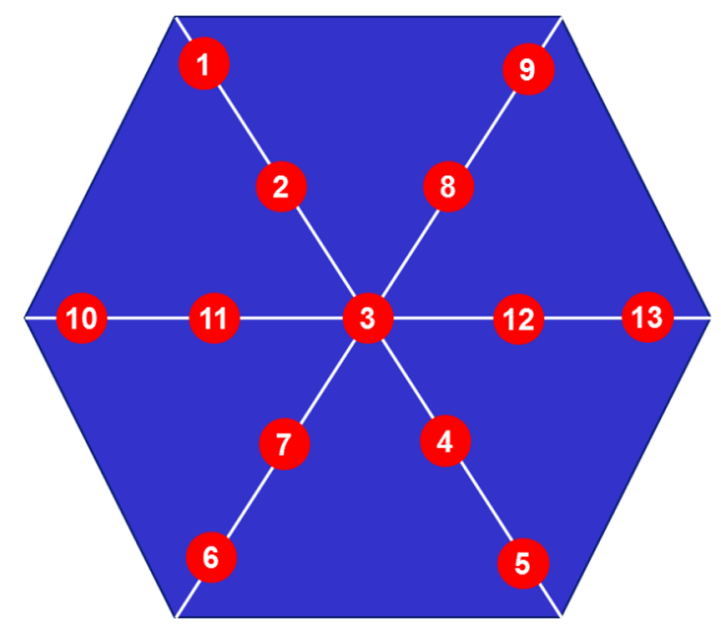

Fig. 3 Distribution of the 13 points used to qualify the mirror reflectivity. 
La Palombara et al.: Mirror production for the Cherenkov telescopes of the ASTRI mini-array...

All these measurements and tests demonstrated that the performance of the produced segments is compliant with the applicable specifications and that the segments do not degrade due to the environmental conditions.

Once the production process was properly qualified, the serial mirror production was approved. It was performed following the production flow reported in Fig. 4.

\section{Quality Assurance Approach}

Once the production process was qualified, in February 2018 it was possible to start with the mirror mass production. In order to further consolidate the manufacturing and verification flowchart of Fig. 4, it has been decided to consider these first sets of panels as a pilot production and to follow a "first article inspection" approach (cf., EN 9102). The panels have been subdued to an extensive set of acceptance verifications at the manufacturer site, double-checked independently for the most critical parameters at the Customers laboratories. The verification approach and the relevant tests for the different sets of mirrors are described in Sec. 6. The obtained results are detailed in Sec. 7.

First, for each mirror segment, a series of acceptance tests and measurements were performed by ML. As a first step, a visual inspection was executed, to ascertain the mirror integrity and evaluate the cosmetic defects (such as scratches, spots, and halos) of the reflective surface. Then a tape removal test was performed, in order to verify the coating adhesion. Afterward, different types of measurements were performed in order to characterize the optical and metrological properties of each segment.

The reflectivity curves were measured in five different equidistant points along one diagonal: two positions are near two opposite segment vertexes and one is at the segment center, whereas the remaining two positions are at intermediate distance between the center and the two opposite vertexes. In this way, the five reflectivity curves provide a representative picture of the whole segment. The average reflectivity and the reflective nonuniformity were derived from these curves.

From the metrological point of view, in the case of the focusing MST segments, the measurement of the RoC and of the PSF size (at both the best and the NF length) was performed. In the case of the aspheric ASTRI M1 segments, instead, the best-fit value of the spherical component and the residual shape error were measured. The accuracy of the RoC measurement performed with the CMM is about $50 \mathrm{~mm}$ and is obtained by spherical best fit of the measured data points. In production, the optical surface is sampled in 536 points. The above uncertainty has been estimated by comparison with the shape of the mirror derived from measurements done with a much finer grid, which is impractical in an industrial environment.

The measurement of the PSF size is obtained with an optical bench, based on a $2 f$ configuration, which generates a spherical wave and detects the focused back-reflected light beam. The set-up of this facility consists of a point-like (with respect to mirror dimension and curvature) laser source at $632 \mathrm{~nm}$ that is converted into a spherical wave by means of a microscope eyepiece. The required numerical aperture is about 0.016 and it is easily achieved. The MST mirror is positioned at a distance of $32,140 \mathrm{~mm}$ with an accuracy of the order of $1 \mathrm{~mm}$. The backreflected radiation illuminates a screen positioned at the focal plane, which is in turn imaged on a $2048 \times 2048$ pixel CCD camera. The camera covers a physical area of $170 \mathrm{~mm} \times 170 \mathrm{~mm}$. In order to avoid superposition between the source and the image plane, each of them is transversally displaced from the optical axis by $100 \mathrm{~mm}$. The measurement of the $D_{80}$ diameter has an accuracy of $3 \mathrm{~mm}$. This is due to several reasons, which include the contribution of variable daylight illumination (despite of the presence of a filter in front of the CCD), the finite area of the focal plane imaged onto the CCD, the scattering of light from the screen. The entity of the accuracy $(3 \mathrm{~mm})$ has been estimated by repeated measurements and comparison with controlled laboratory conditions, which of course are not completely possible in an industrial environment.

Apart from the acceptance tests performed on each segment, additional tests were performed by ML on some individual segments regarding the microroughness and the water tightness. These tests were necessary in order to keep under control the production process. 
La Palombara et al.: Mirror production for the Cherenkov telescopes of the ASTRI mini-array...

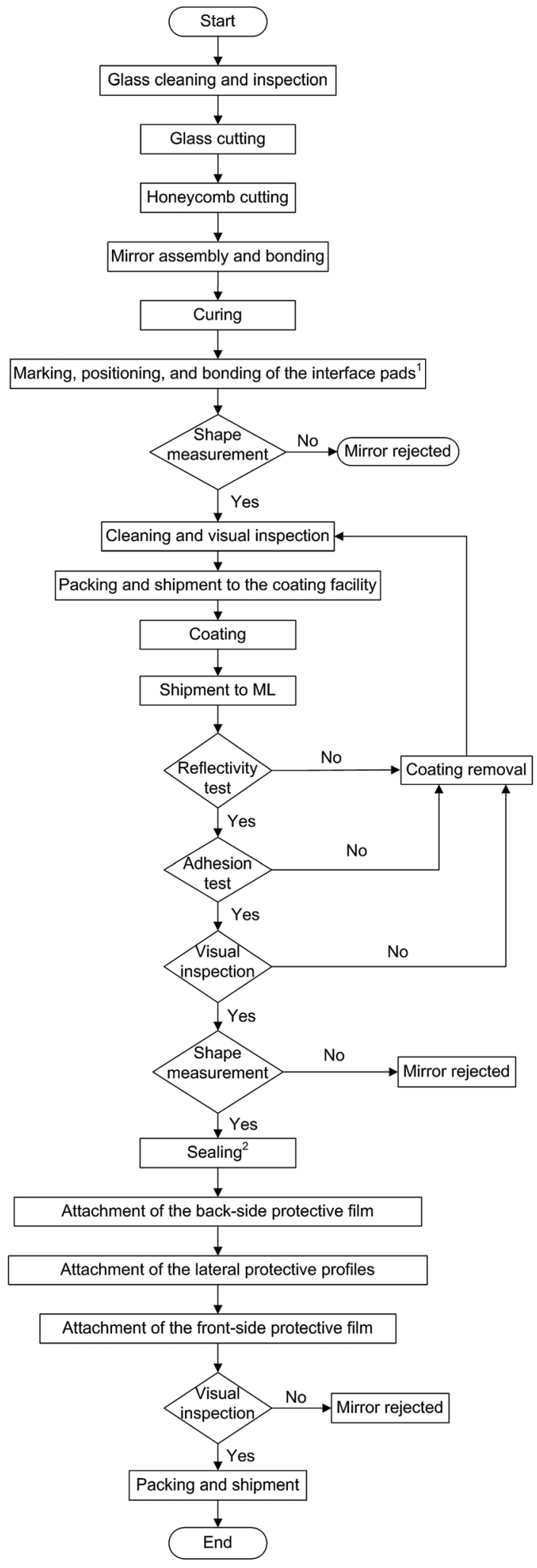

Fig. 4 Production flow for the ASTRI M1 and MST mirrors. Notes: (1) Three pads are installed on each mirror to provide mechanical interface with the telescope mechanical structure. (2) The lateral surfaces of each mirror are sealed with silicone to prevent the penetration of atmospheric agents. 
The contract assigned to ML requires that each mirror segment is associated with an "identity card" (IC), which reports the results of all the acceptance tests and measurements. The IC is the reference document to control the mirror quality. Along the production phase, the quality assurance team used it to monitor the production process and decide which segments required a direct inspection, in order to evaluate if they can be accepted or rejected. In case a segment showed a major anomaly (see Fig. 5), which did not affect its performance or reliability (such as a scratch or a wide halo), it was accepted only if a comprehensive description of the anomaly was reported in a nonconformance report (NCR). If, on the other hand, the detected anomaly compromised the segment performance or reliability (such as a large spalling of the mirror edge), the segment was rejected. After its delivery, each segment must be followed by its IC (and the relevant NCRs, if any) for all its life. In this way, at any time it will be possible to retrieve the mirror properties and evaluate if it is suitable for the installation on the telescope.

Additional sample tests were performed by OAB and DESY at their laboratories on $\sim 10 \%$ of the produced mirrors. Such tests concerned the RoC and PSF measurement for the MST segments and the shape measurement for the ASTRI M1 segments. In this way, we obtained an independent check of the segment properties, monitored the reliability of the production process, and provided feedback to the industrial supplier.

The 200 MST segments were delivered by the industrial supplier in 4 different batches of $\sim 50$ segments each. For each of these batches, we selected 5 segments $(\sim 10 \%$ of the whole batch) for additional measurements. In all cases, the 5 segments were selected as follows: 2 among the segments with the best PSF (i.e., small $D_{80}$ ); 2 among the segments with the worst PSF (i.e., large $D_{80}$ ); 1 segment with intermediate PSF. We made such selection in order to check whether the results obtained at ML and those obtained with additional measurements depend on the mirror shape.

For 15 of these MST segments, the additional measurements were performed at INAF-OAB, using an outdoor optical bench based on a $2 f$ configuration, installed at the laboratories of the Brera Astronomical Observatory in Merate (Italy). ${ }^{55}$ Other 5 MST segments (different from those measured at OAB) were instead verified at the DESY $2 f$ optical bench. ${ }^{56}$ In both cases, the $2 f$ facility uses a LED as light source (without any diffuser, since the generated wavefront is already spherical) and a CCD camera as image detector: in this way a photometric image is obtained, which is characterized by high homogeneity and uniformity.

For each tested MST segment, the following measurements were performed.

- Mirror RoC performed by acquiring a series of images of the focal spot along the optical axis.

- Mirror $D_{80}$ at both the NF length, corresponding to the nominal $2 f$ value of $32.14 \mathrm{~m}$, and the best focal (BF) length, which is the $2 f$ value where the $D_{80}$ value is minimum

(a)

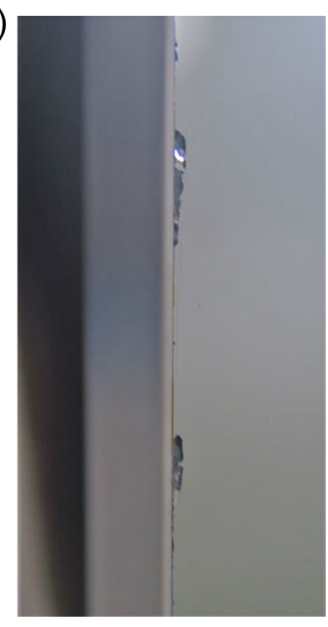

(b)

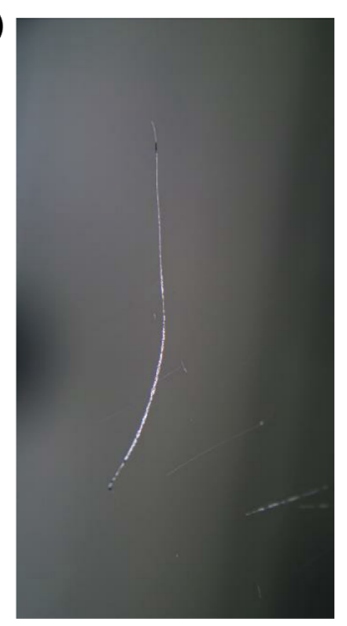

Fig. 5 Examples of the most common mirror defects: (a) edge irregularities and (b) coating scratches. 
The final measurement regarded the focused reflectivity of the MST segments. In fact, while the average reflectivity reported in the IC of each segment is the average of the surface reflectivity measured at five different positions along one diagonal, the focused reflectivity is the reflectivity of the overall segment measured at the focal plane. Therefore, it is a parameter which truly represents the CTA requirement. The measurement of this parameter has been performed at the $2 f$ UV-CCD facility of the Institut für Astronomie und Astrophysik (IAAT) of the University of Tübingen (Germany), ${ }^{57}$ using the same five MST segments which were verified at DESY.

\section{Results}

\subsection{Overview}

The mirror production was completed at the end of 2019. The whole set includes 200 MST segments and 200 ASTRI M1 segments (68 COR1, 66 COR2, and 66 COR3). The MST segments correspond to two full mirrors (of 86 segments each) plus 28 spare segments. In the case of ASTRI M1, the delivered segments correspond to 11 complete M1 mirrors (of $6 \times 3$ segments each) plus two additional COR1 segments for test purposes.

\subsection{Results for MST Segments}

\subsubsection{Results obtained at $M L$}

In Fig. 6, we show the main parameters of the MST segments, as reported in the relevant ICs delivered by the industrial supplier. In (a), the reference nominal value is represented with a green solid line, whereas in (b)-(d), the applicable requirement value is represented with a red solid line. In (a)-(d), the average value is represented with a black dashed line. The colored regions above and below the average value correspond to 1, 2, and 3 standard deviations $(\sigma)$ from the average value, respectively.

(a)

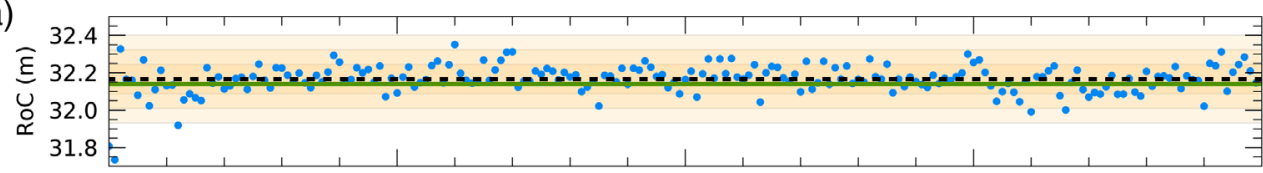

(b)

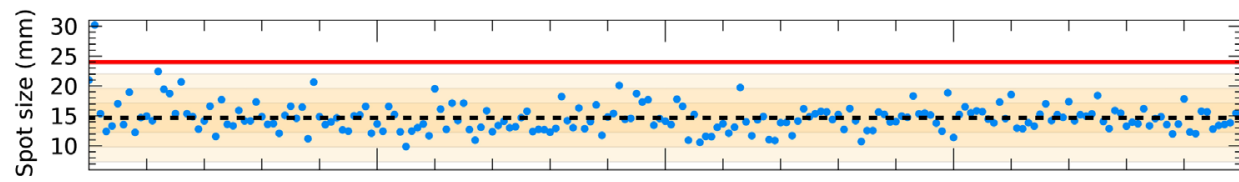

(c)

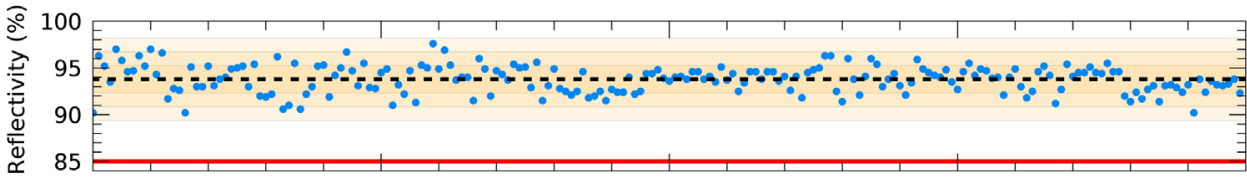

(d)

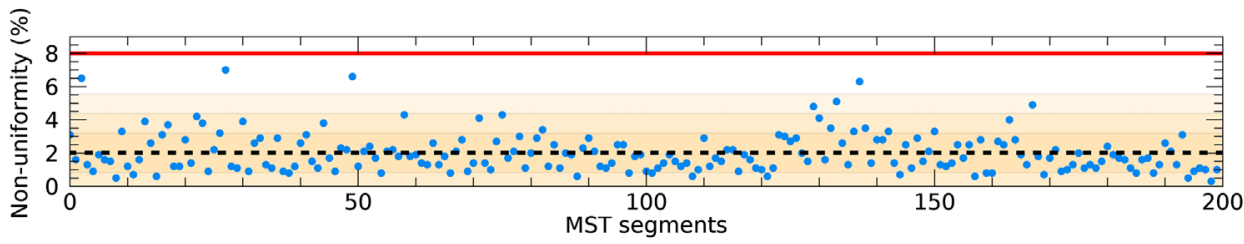

Fig. 6 Main parameters of the produced MST segments, from the first (segment 0 ) to the last made (segment 199): (a) radius of curvature, (b) $D_{80}$ at twice the NF length, (c) average reflectivity, and (d) reflectivity nonuniformity. In (a), the green solid line represents the reference nominal value; whereas in (c)-(d), the red solid line represents the applicable requirement value. In (a)-(d), the black dashed line represents the average value. 
Figure 6(a) reports the measured value of the RoC. For almost all the segments, this value is close to the nominal value of $32.14 \mathrm{~m}$ (although in many cases it is not within the range 32.10 to $32.18 \mathrm{~m}$ ) and shows no tendency to increase or decrease. The only exceptions regard the first two segments, which have a significantly smaller radius. They were produced when the process was not yet completely assessed. This means that, after these first two segments, the production process has remained stable. In any case, it should be noted that the requirement on the RoC is not considered an applicable acceptance criterion, since what matters is only the value of $\theta_{80}$ at the NF length. On the other hand, for all but one of the segments produced so far, the $D_{80}$ value was lower than $24 \mathrm{~mm}$ at twice the focal length [at $2 f$, Fig. 6(b)]: this implies that these mirrors are considered compliant with the CTA requirement of $D_{80}<12 \mathrm{~mm}$ at $1 f$. Moreover, apart from this isolated worst case, the measured data show no long-term increasing or decreasing trend in the production. The average reflectivity [Fig. 6(c)] is well above $85 \%$ for all the segments, and the average reflectivity nonuniformity of the mirrors [Fig. 6(d)] is always below the requirement of $8 \%$. Therefore, also these parameters are compliant with the corresponding input requirements.

In Fig. 7, the previous results are summarized in histograms, which report the distribution of each parameter around its average value. They show that the distribution is rather symmetric for the RoC and the reflectivity, while it has a tail toward the high values for $D_{80}$ and the reflectivity nonuniformity.

\subsubsection{Results of sample tests performed at INAF-OAB and DESY}

In Fig. 8, we report, for each of the 20 sample segments that were independently double-checked, the difference between the RoC values measured at the CMM in ML (those reported in the

(a)

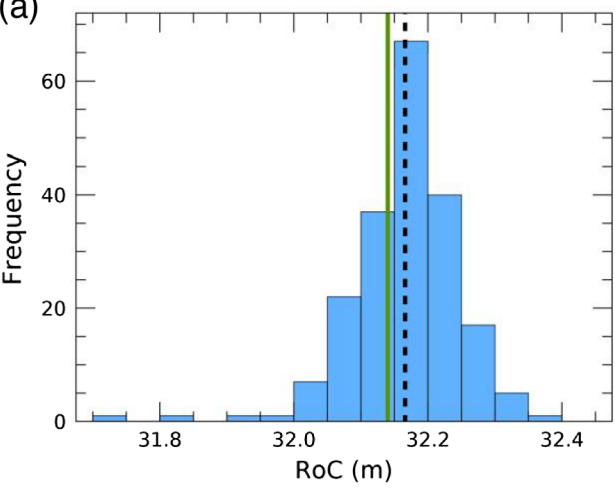

(c)

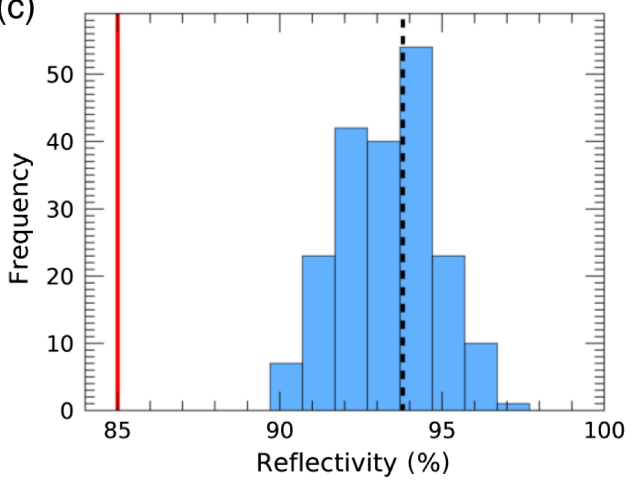

(b)

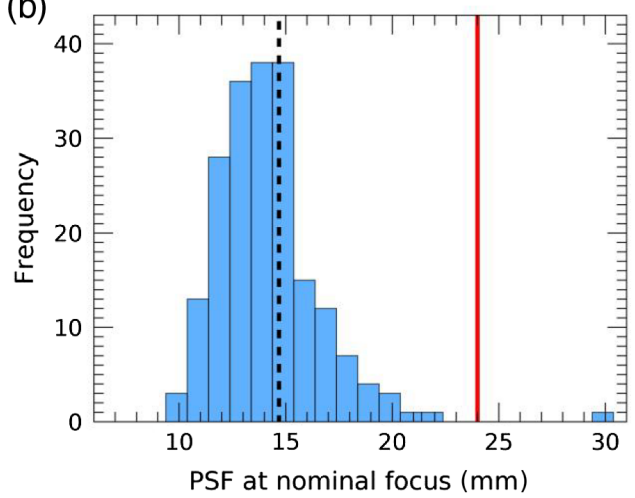

(d)

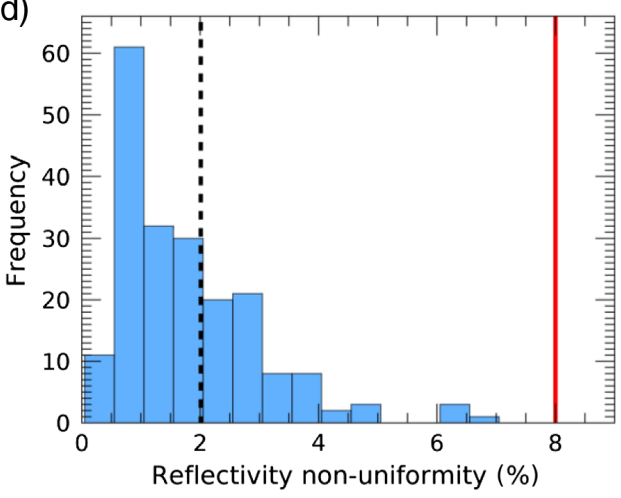

Fig. 7 Distribution of the main parameters for the MST segments: (a) radius of curvature, (b) $D_{80}$ at twice the NF length, (c) average reflectivity, and (d) reflectivity nonuniformity. In (a), the green solid line represents the reference nominal value; whereas in the other panels, the red solid line represents the applicable requirement value. In all panels, the black dashed line represents the average value. 


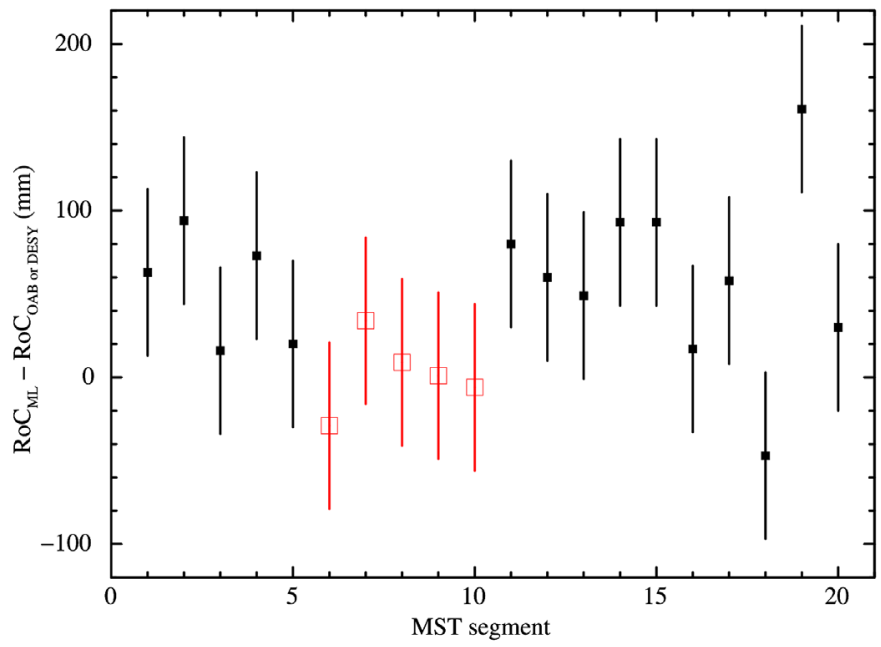

Fig. 8 Difference between the mirror radii of curvature measured at ML and either OAB (filled black squares) or DESY (empty red squares). The error bars indicate the systematic error of the CMM.

segment ICs) and the corresponding values measured at either the OAB or the DESY $2 f$ facility. It shows that, while there is no systematic difference between the results obtained at OAB and DESY, for most segments the RoC measured by ML is larger than that obtained with the other facilities. This difference is likely due to the different segment positions during the measurement: in fact, the segment lies horizontally during the measurement with the ML CMM, while it is mounted vertically at the $2 f$ facility. This implies an opposite impact of the gravity, which increases the $\mathrm{RoC}$ in the first case and reduces it in the second one.

Taking into account these points, the values measured at OAB and DESY are compatible with those provided by ML.

In Fig. 9, we report, for each segment, the difference between the $D_{80}$ measured by ML and the corresponding value measured at either the OAB or the DESY $2 f$ facility, at both best and NF length. It shows that also in this case the results obtained by OAB and DESY are compatible. On the other hand, at both the BF and NF positions, for most segments the $D_{80}$ value obtained at $\mathrm{OAB}$ or DESY is slightly lower than the corresponding value obtained by ML. This difference is due to the different methods used by ML and OAB or DESY to perform the $D_{80}$ measurement. In fact, the measurements performed at OAB and DESY use a LED as light source (without any diffuser, since the generated wavefront is already spherical) and a CCD camera as image detector: in this way, a photometric image is obtained, which is characterized by high homogeneity and uniformity.
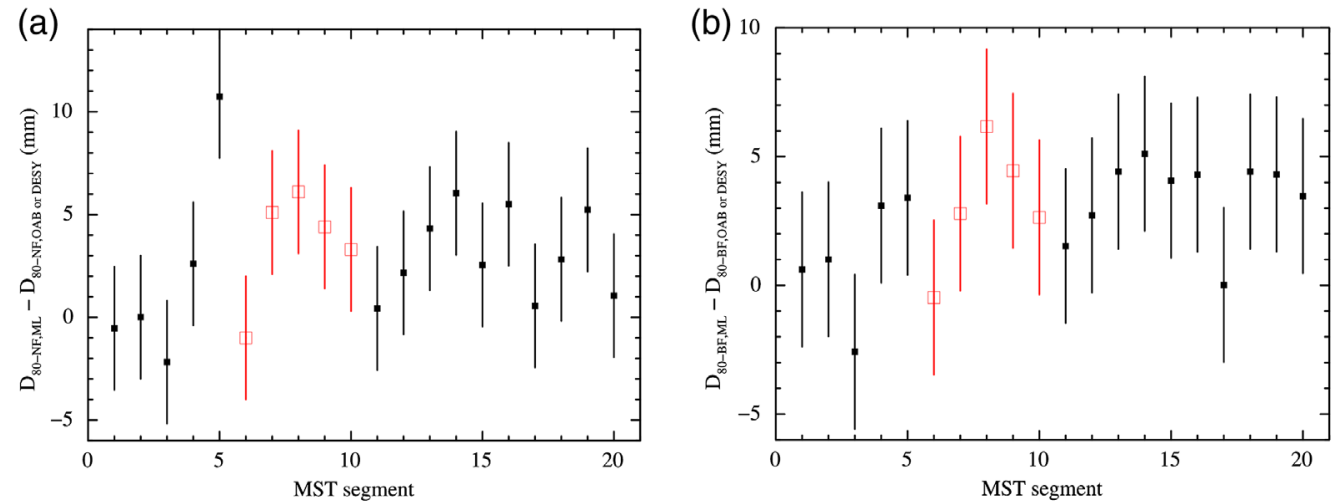

Fig. 9 Difference of the $D_{80}$ measurements at ML and either OAB (filled black squares) or DESY (empty red squares) at (a) nominal focus and (b) best focus. The error bars indicate the systematic error of the ML measurements. 
These differences in the measurement approach explain the larger $D_{80}$ value obtained (on average) by ML, compared with that obtained at OAB and DESY. Taking into account the intrinsic uncertainty of these measurements, the two methods provide results that can be considered compatible; the results just confirm that $D_{80}$ is well within the specification for all segments also using a different configuration for the characterization.

\subsubsection{Results of sample tests performed at IAAT}

The $2 f$ principle assumes a point-like light source, which is realized at IAAT with LEDs of different wavelengths $(310,365,405,490$, and $525 \mathrm{~nm})$ driven by a highly stable current supply. A spherical mirror placed at a distance equal to its RoC, i.e., two times its focal length $f$, generates an image of the light source at the same distance. We project this image on a diffuse polytetrafluoroethylene screen and record it with a UV-sensitive CCD camera.

The focused reflectivity of the MST segments was determined by integrating the reflected intensity over a circular area with $2 \mathrm{mrad}$ radius around the center of the spot and dividing by the incident intensity on the mirror. The incident intensity was measured by placing the screen between light source and mirror and integrating over the projected mirror area. Moreover, the same data were used to determine the mirror PSF, which was then averaged over the five wavelengths. $100 \%$ of the intensity for the $D_{80}$ determination was defined as the intensity in a circular area with $2 \mathrm{mrad}$ radius around the center of the spot. This angular scale refers to the $2 f$ distance of the measurement, thus in the MST case with a nominal RoC of $32.14 \mathrm{~m}, 2 \mathrm{mrad}$ corresponds to a radius of about $64 \mathrm{~mm}$.

In Table 2, we report, for each of the five MST segments, the focused reflectivity at each of the five wavelengths and the average value of $D_{80}$. It shows that, for all mirrors, the reflectivity reaches $85 \%$ at all wavelengths and the $D_{80}$ value is $\leq 14 \mathrm{~mm}$, in agreement with the input requirements.

\subsubsection{Summary on MST results}

In Table 3, we report the summary of the results obtained with the various metrology measurements performed on the MST segments. For all sets of segments, the average RoC is in the range 32.1 to $32.2 \mathrm{~m}$, with a standard deviation of 5 to $10 \mathrm{~cm}$ around the average value. This means that, in all cases, the average RoC is very near the nominal value of $32.14 \mathrm{~m}$, and that the dispersion around this value is very low. Regarding the PSF size, for all sets the average $D_{80}$ value is in the range 11 to $15 \mathrm{~mm}$, with a standard deviation of 1 to $3 \mathrm{~mm}$ around the average value. Therefore, also in the case of the PSF size, all measurements confirm that the produced segments are well within the requirement of $24 \mathrm{~mm}$ at $2 f$.

In Table 4, we report instead the summary of the reflectivity measurements performed at ML (for the surface reflectivity) and at IAAT (for the focused reflectivity). It shows that, in both cases, the average reflectivity value is well above the requirement of $85 \%$ and the standard

Table 2 Focused reflectivity and PSF spot size measured at IAAT.

\begin{tabular}{lcccccc}
\hline \hline & \multicolumn{5}{c}{ Reflectivity (\%) } \\
\cline { 2 - 5 } Mirror & $310 \mathrm{~nm}$ & $365 \mathrm{~nm}$ & $405 \mathrm{~nm}$ & $490 \mathrm{~nm}$ & $525 \mathrm{~nm}$ & $D_{80}(\mathrm{~mm})$ \\
\hline MST-ML-114 & $85 \pm 2$ & $88 \pm 2$ & $89 \pm 2$ & $86 \pm 2$ & $86 \pm 2$ & $12.5 \pm 0.2$ \\
MST-ML-118 & $88 \pm 2$ & $92 \pm 2$ & $94 \pm 2$ & $91 \pm 2$ & $91 \pm 2$ & $14.0 \pm 0.3$ \\
MST-ML-122 & $88 \pm 2$ & $90 \pm 2$ & $91 \pm 2$ & $88 \pm 2$ & $88 \pm 2$ & $13.4 \pm 0.2$ \\
MST-ML-123 & $85 \pm 2$ & $91 \pm 2$ & $92 \pm 2$ & $91 \pm 2$ & $89 \pm 2$ & $12.3 \pm 0.3$ \\
MST-ML-124 & $85 \pm 2$ & $90 \pm 2$ & $91 \pm 2$ & $88 \pm 2$ & $88 \pm 2$ & $14.0 \pm 0.1$ \\
\hline \hline
\end{tabular}







La Palombara et al.: Mirror production for the Cherenkov telescopes of the ASTRI mini-array...

Table 4 Summary of the reflectivity results obtained for the MST segments.

\begin{tabular}{|c|c|c|c|c|}
\hline Laboratory & $\begin{array}{l}\text { Measured } \\
\text { parameter }\end{array}$ & Measuring tool & $\begin{array}{c}\text { Number of } \\
\text { measured } \\
\text { segments }\end{array}$ & Results \\
\hline ML & $\begin{array}{l}\text { Surface } \\
\text { reflectivity }\end{array}$ & $\begin{array}{l}\text { Measurement on five different points } \\
\text { using a thin-film analyzer }(\lambda=190 \text { to } \\
1700 \mathrm{~nm}) \text { with a contact probe and } \\
\text { deuterium-tungsten light source }\end{array}$ & 200 & $\begin{array}{l}\text { Average value: } 93.8 \% \\
\text { Standard deviation: } 1.5 \%\end{array}$ \\
\hline $\begin{array}{l}\text { IAAT } \\
\text { Tubingen }\end{array}$ & $\begin{array}{l}\text { Focused } \\
\text { reflectivity }\end{array}$ & $\begin{array}{l}2 f \text { facility with a LED source and a } \\
\text { CCD camera }\end{array}$ & 5 & $\begin{array}{l}\text { Average value: } 89.0 \% \\
\text { Standard deviation: } 1.6 \%\end{array}$ \\
\hline
\end{tabular}

deviation around it is below $2 \%$. This result confirms the high stability reached with the adopted production process.

These results show that the measurements of the RoC and $D_{80}$ performed on the MST segments with different facilities provided compatible values and give us high confidence on both the reliability of the production process and the compliance of the mirrors with the CTA requirements.

\subsection{Results for ASTRI M1 Segments}

Regarding the ASTRI M1 segments, in this section, we report the results obtained for all the 68 COR1 segments, while in the Appendix, we report the corresponding results for COR2 and COR3 segments. In all cases, they are retrieved from the relevant ICs delivered by ML.

In Figs. 10(a) and (b), we show, respectively, the values of the RoC and the residual shape errors. (We remind here that the ASTRI M1 segments are aspherical and that RoC refers to the spherical component.) For all COR1 segments, the first parameter is within the required range (between 8198 and $8298 \mathrm{~mm}$ ) and the second parameter is <20 $\mu \mathrm{m}$ RMS, in agreement with

(a) 8.35

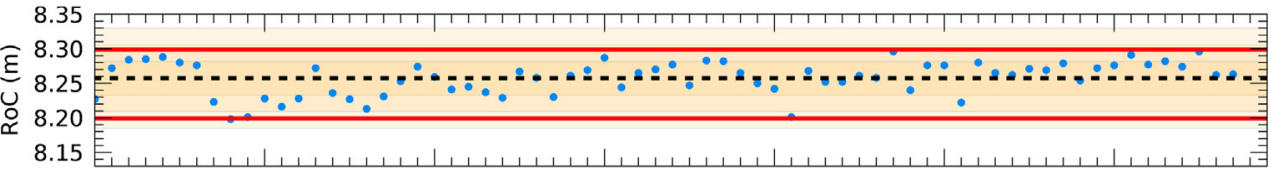

(b)

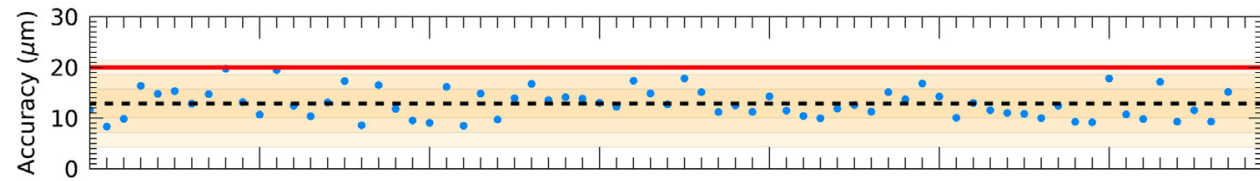

(c)

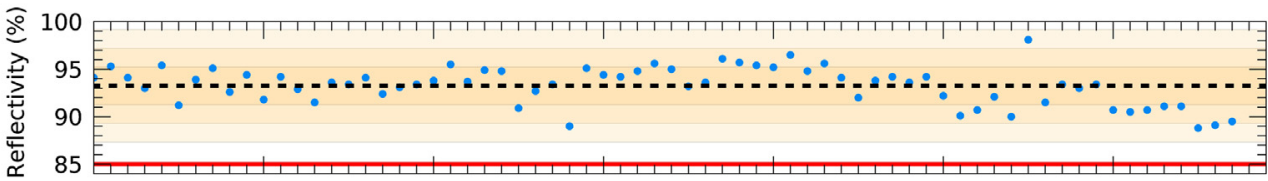

(d)

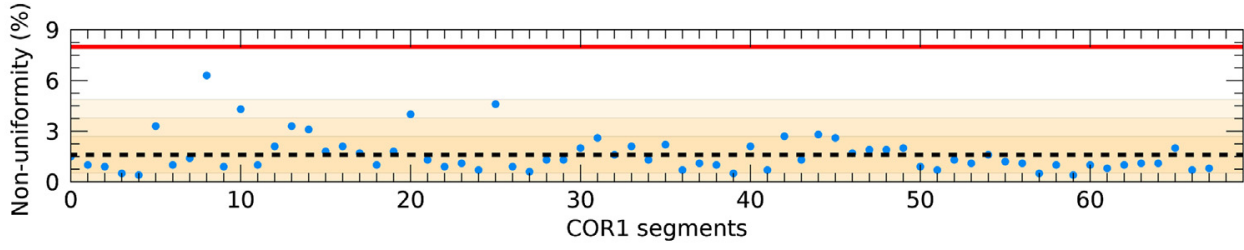

Fig. 10 Main parameters of the COR1 segments of ASTRI M1 from the first (segment 0) to the last made (segment 67): (a) radius of the spherical component, (b) residual shape error, (c) average reflectivity, and (d) reflectivity nonuniformity. For each panel, the solid red and dashed black lines represent, respectively, the applicable requirement value and the average value. 
(a)

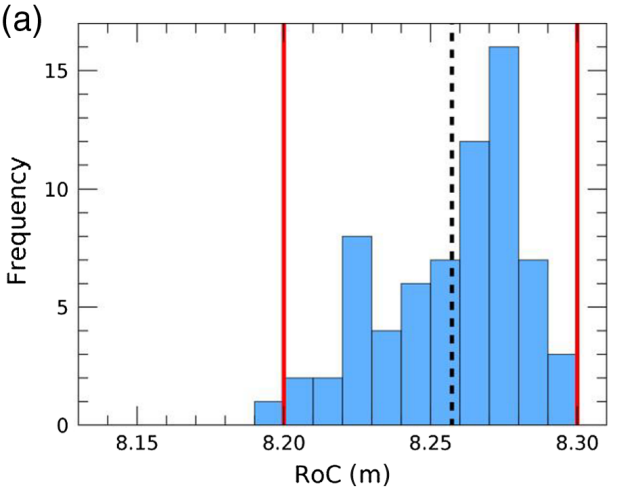

(c)

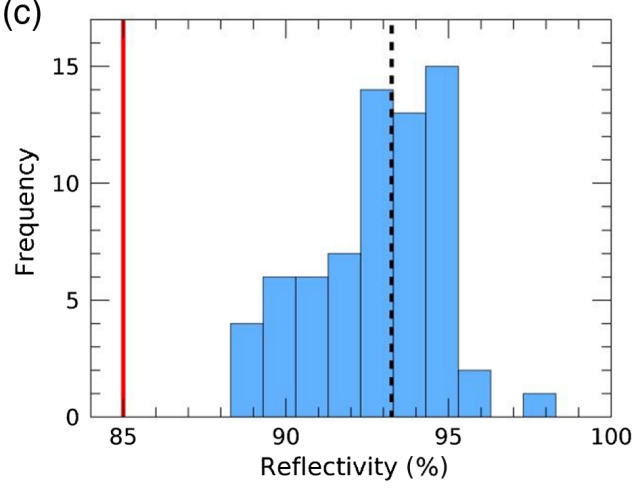

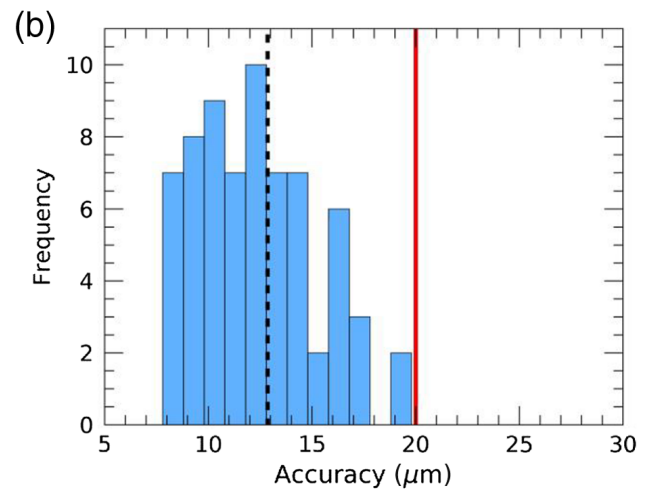

(d)

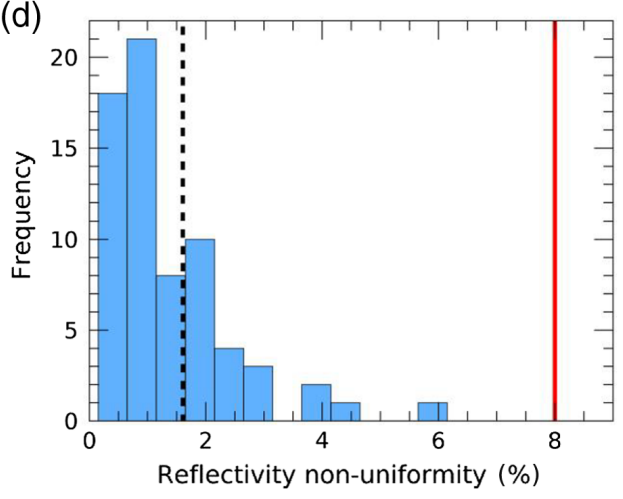

Fig. 11 Distribution of the main parameters for the COR1 segments: (a) radius of curvature, (b) residual shape error, (c) average reflectivity, and (d) reflectivity nonuniformity. For each panel, the red solid and black dashed lines represent, respectively, the applicable requirement value and the average value.

the corresponding requirement. Regarding the mirror reflectivity, in the Figs. 10(c) and (d) we report, respectively, its average value and its nonuniformity for each segment. As for the MST segments, also for all the ASTRI COR1 segments, these parameters are compliant with the lower limit of $85 \%$ and the upper limit of $8 \%$, respectively.

In Fig. 11, the previous results are summarized in histograms, which report the distribution of each parameter around its average value. They show that, for all the COR1 segments, the values of RoC and accuracy have a wide and rather uniform distribution around the average value, while the distribution of the reflectivity values is rather peaked around the average value. Regarding the reflectivity nonuniformity, most of the segments have a low value, but the overall distribution shows a tail at high values.

In Fig. 12, we show one example of the five reflectivity curves measured for each segment at five equidistant positions along a diagonal. Although these curves differ from each other, all of them are always above the required value of $85 \%$ between 300 and $550 \mathrm{~nm}$, while they decrease at shorter wavelengths, where the data are reported only for information. They have a maximum at about $360 \mathrm{~nm}$, while they show a gradual decrease at longer wavelengths. It should be noted that, in the case of ASTRI, the concept of focused reflectivity is not applicable, since we are talking of a dual-mirror system with the segments of the primary not having a real focus. Finally, in Fig. 13, we report the residual map obtained by comparing the theoretical and the real shapes of one ASTRI M1 segment. It is used to measure the surface accuracies reported in Fig. 10(b).

\section{Summary and Conclusions}

Before starting the mass production, the manufacturing process of the mirror segments, based on the cold replication process set-up by INAF and ML, has been fully qualified for MST and 


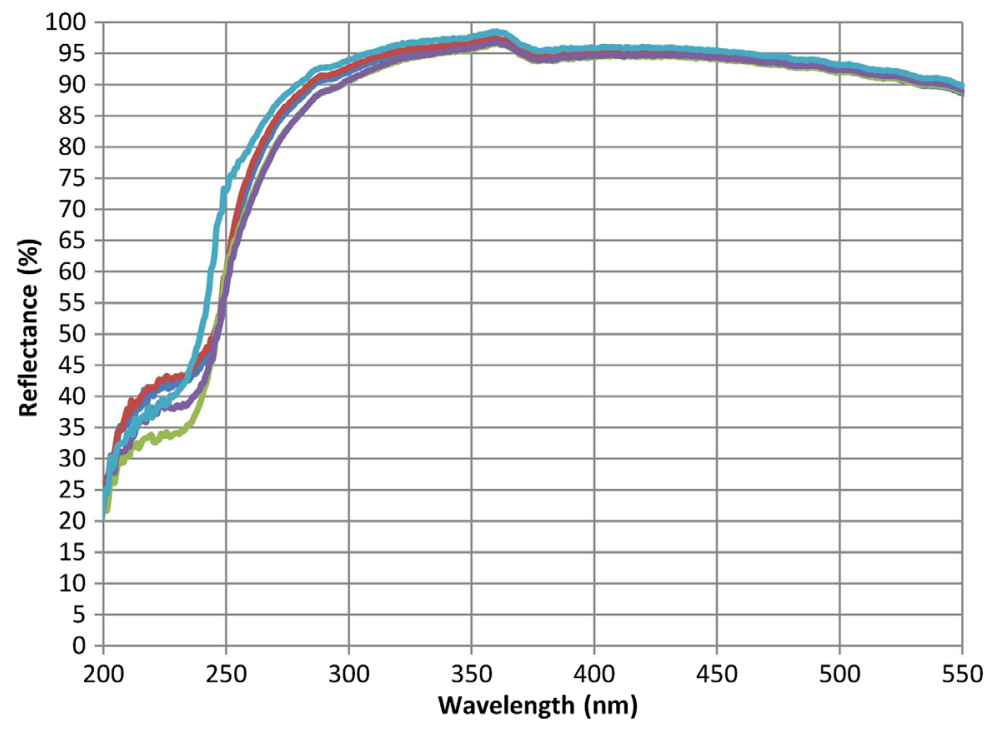

Fig. 12 Example of the reflectivity curves of one ASTRI M1 segment measured at five different positions along a diagonal.
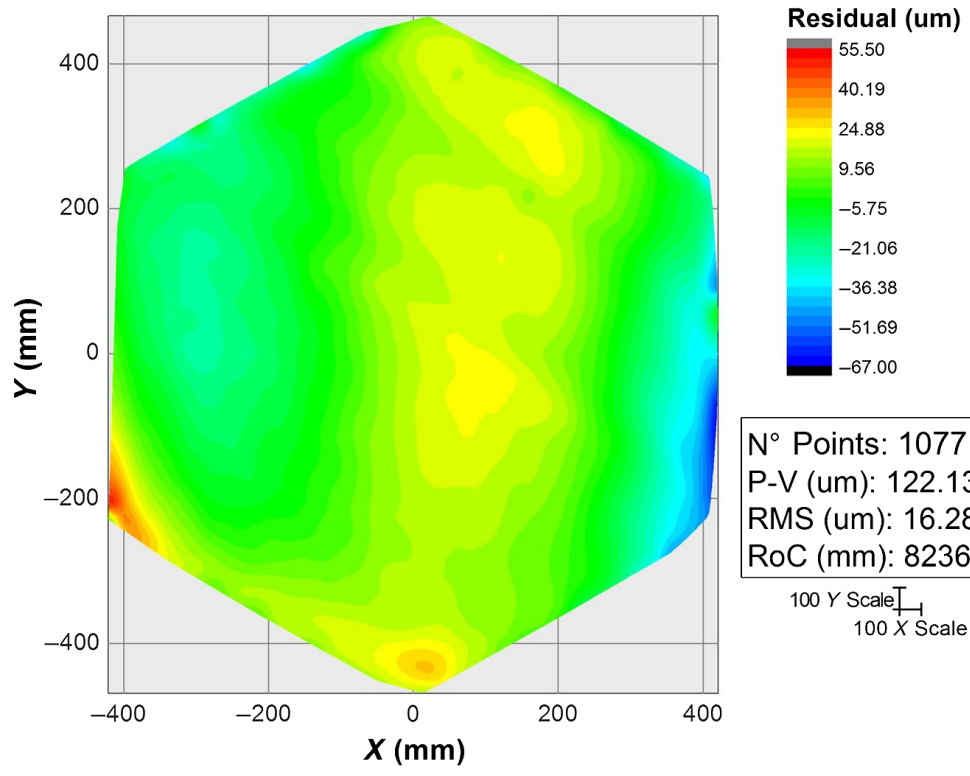

No Points: 1077

P-V (um): 122.13

RMS (um): 16.28

$\operatorname{RoC}(\mathrm{mm}): 8236$

$100 Y$ Scale $T$

$100 \times$ Scale

Fig. 13 Example of the residual map of one ASTRI M1 segment.

ASTRI M1 mirrors, in the context of the CTA and ASTRI MA development phases. The ASTRI MA telescopes also represent the baseline design for the SST telescopes of CTA, which will be just slightly upgraded. It should be reminded that INAF will provide the entire set of mirrors of the 9 MSTs and the 37 SSTs for the CTAO northern and southern sites, respectively, with about 1600 segments to be produced.

In the present work, it has been verified that the developed manufacturing process ensures the realization of mirror segments that are fully compliant with the CTA requirements from the environmental, reliability, and performance points of view. We have also implemented an effective quality assurance approach that allows us to monitor the stability and reliability of the manufacturing process along the mass production.

The mirror production was completed at the end of 2019, with the delivery of 200 MST segments and 200 ASTRI M1 segments fully compliant with the requirements. Since a set of 10 ASTRI M2 mirrors is already available, not only more than enough mirrors are ready 
for the upcoming realization of the ASTRI MA, but a mass production exercise has been successfully carried out in view of the production of the mirrors for the SST CTAO array. In this respect, as the mirrors were highly aspherical, the optical performance was verified by means of metrological measurements, while the reflectivity performance was successfully verified with local measurements on different positions on the surface of each segment.

It should be noted that, in order to evaluate the optical performance of the MST mirrors, the qualification was carried out at the production premises of ML using both metrological and optical measurements. Moreover, other optical verifications were performed on a limited number of mirrors using other external facilities operated by INAF, DESY, and IAAT. All the results achieved are in good agreement. The reflectivity was measured on local areas of each mirror and, for a limited number of pieces, also in terms of focused reflectivity. Once again, the results are in good accordance. This means that the production process is stable and ensures the production of mirrors conforming to specifications. Taking into account the systematic effects of the different tests, the measurements carried out independently on sample mirrors confirmed the results obtained at the production site. Therefore, the results obtained with the described pilot production confirm that the acceptance criteria adopted by the industrial supplier are reliable. Based on this result, the current baseline for the future productions is to maintain the standard set of acceptance tests carried out at ML (reported in the flowchart of Fig. 4), with the only exception of the metrological measurements performed with the CMM. Since this type of measurements is particularly time-consuming, they will be performed only on sample mirrors, in order to monitor the production process. On the other hand, we will still perform independent verifications at $\mathrm{OAB}$ and DESY laboratories, but on a reduced percentage of mirrors compared with the $\sim 10 \%$ adopted for the pilot production.

\section{Appendix: Results for ASTRI COR2 and COR3 Segments}

In Figs. 14 and 15, we report the results obtained for, respectively, the ASTRI COR2 and COR3 segments.

(a)

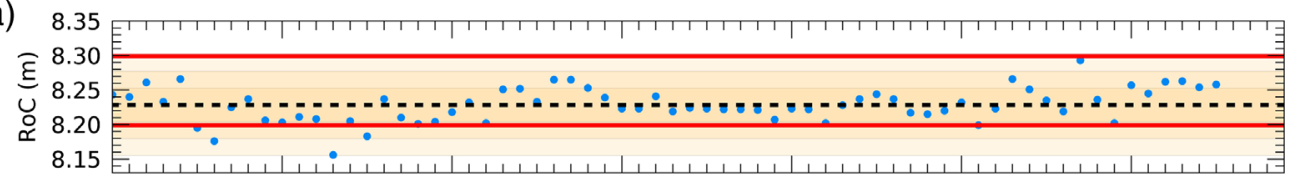

(b)

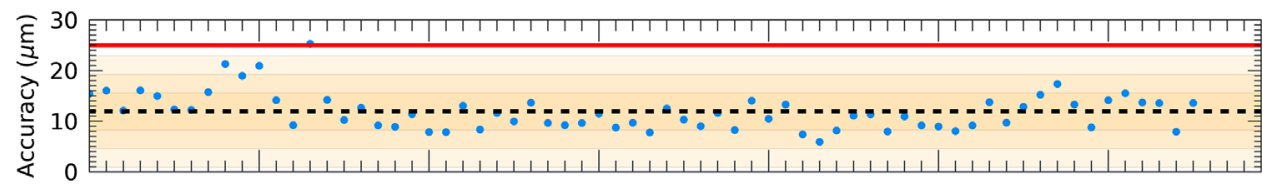

(c)

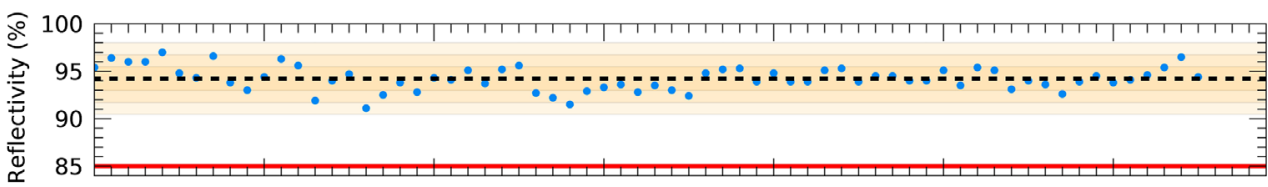

(d)

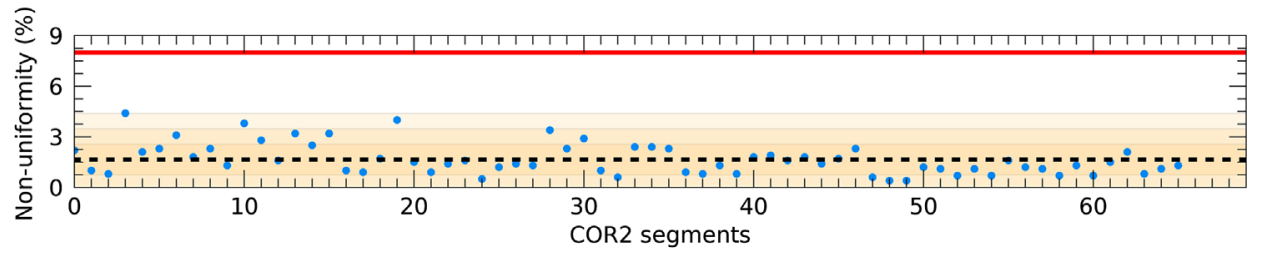

Fig. 14 Main parameters of the COR2 segments of ASTRI M1 from the first (segment 0) to the last made (segment 65): (a) radius of the spherical component, (b) residual shape error, (c) average reflectivity, and (d) reflectivity nonuniformity. For each panel, the solid red and dashed black lines represent, respectively, the applicable requirement value and the average value. 
(a) 8.35

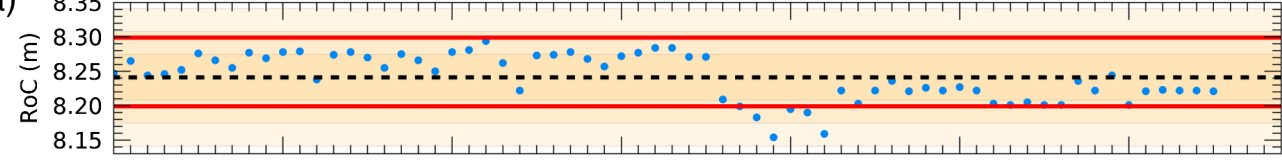

(b)

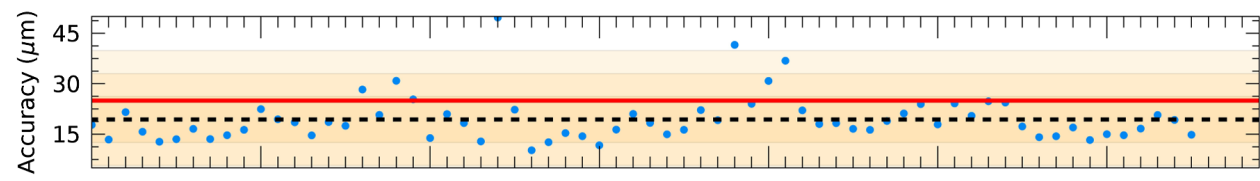

(c)

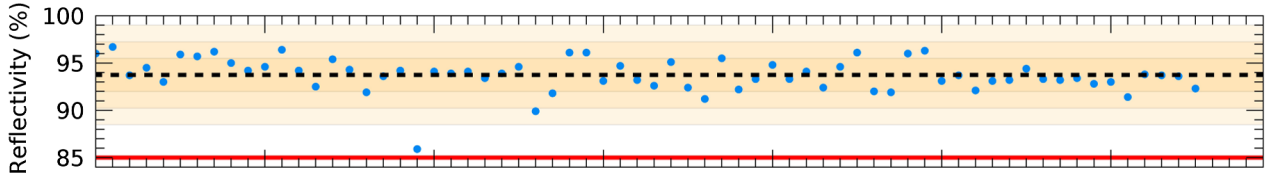

(d)

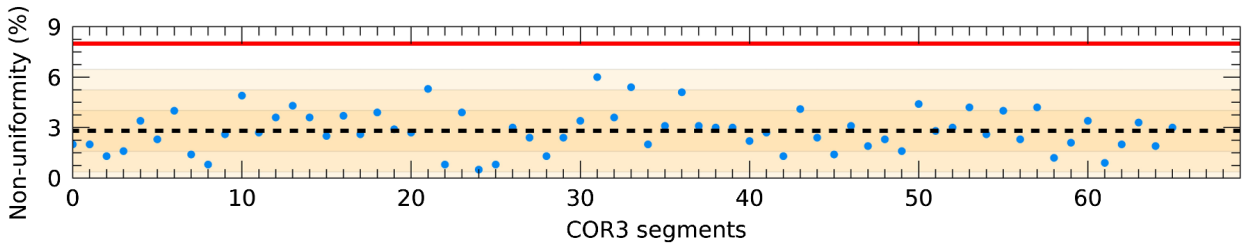

Fig. 15 Main parameters of the COR3 segments of ASTRI M1 from the first (segment 0) to the last made (segment 65): (a) radius of the spherical component, (b) residual shape error, (c) average reflectivity, and (d) reflectivity nonuniformity. For each panel, the solid red and dashed black lines represent, respectively, the applicable requirement value and the average value.

(a)

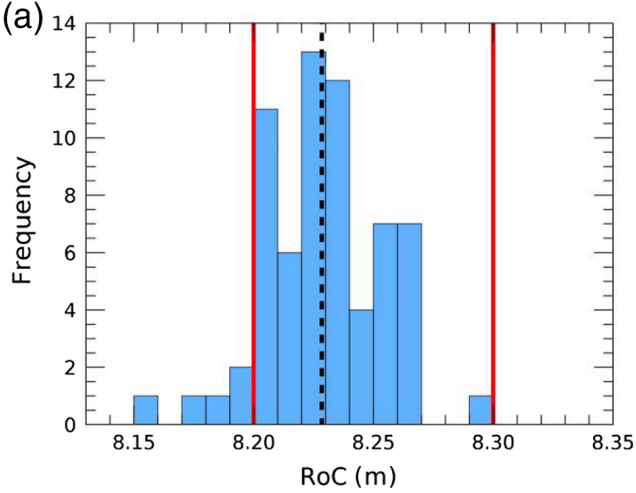

(c)

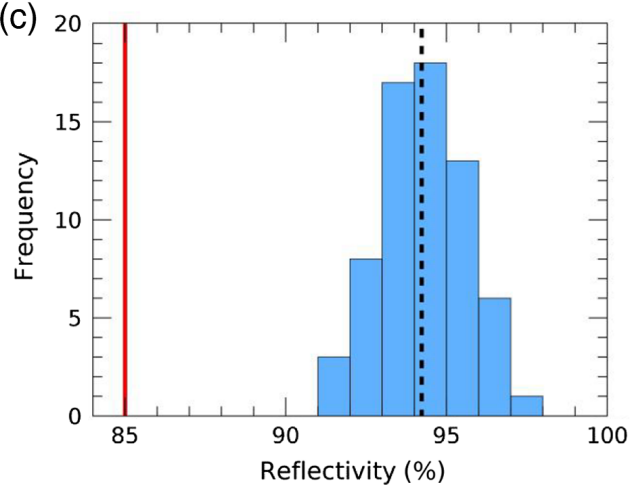

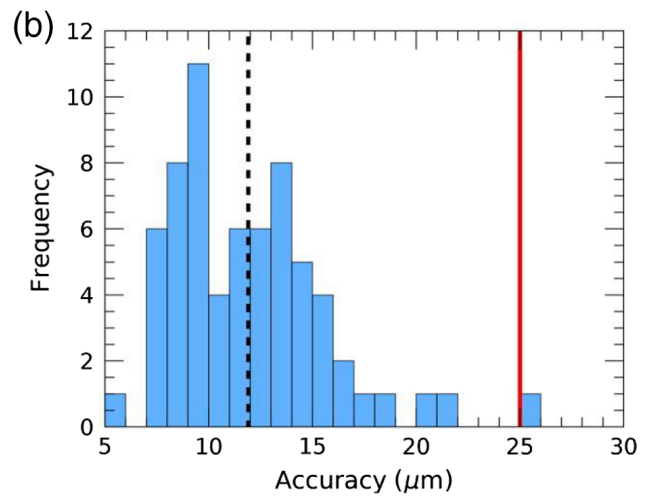

(d)

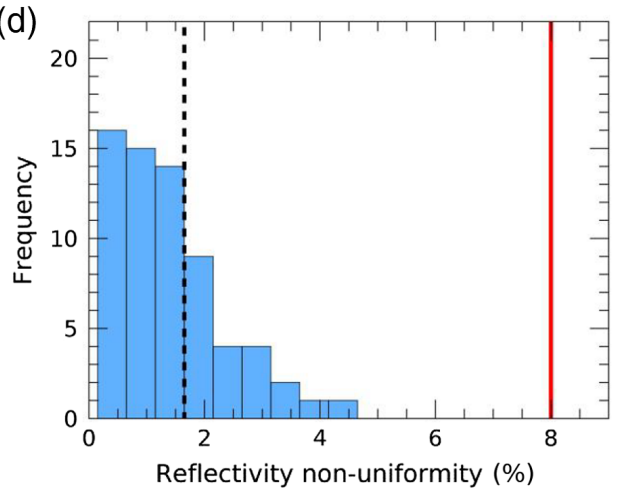

Fig. 16 Distribution of the main parameters for the COR2 segments: (a) radius of curvature, (b) residual shape error, (c) average reflectivity, and (d) reflectivity nonuniformity. For each panel, the red solid and black dashed lines represent, respectively, the applicable requirement value and the average value. 
In Figs. 14(a),(b) and 15(a),(b) we show, respectively, the values of the RoC and the residual shape errors. The first parameter is within the required range (between 8198 and $8298 \mathrm{~mm}$ ) for nearly all the segments. The only exceptions are 4 COR 2 segments and 5 COR 3 segments, for which $\mathrm{RoC}<8198 \mathrm{~mm}$. Regarding the COR3 segments, we can note that the first 35 segments have an $\mathrm{RoC}$ systematically larger than the following ones. This change is due to the replication mould, which was reworked after the production of the first 35 segments. The second parameter is $>25 \mu \mathrm{m}$ RMS for 1 and 7 segments, respectively. Moreover, regarding the COR2 segments, we can note that the residual shape error of the first 13 segments is larger than for the other segments, where the accuracy improved thanks to a better tuning of the replica process. For the segments which are not compliant with the requirement on the $\mathrm{RoC}$ and/or the residual shape error, it was necessary to perform a ray-tracing analysis of the shape data in order to assess that the segment shape is correct. In fact, even mirrors characterized by residual shape errors larger than the requirement can fulfill the telescope optical performance requirement. This is possible because the telescope optical performance requirement is defined on the angular dimension of the PSF across the field of view, whereas the requirement on the residual shape error is an extrapolation of such definition to mirror shape error heights. The measured shape of (a)-(d) out of specification was used as input to simulate with a ray-tracing code the PSFs of the (a)-(d) at the focal plane, assuming the ASTRI design for the secondary mirror and detector. In this way, we evaluated the optical performance degradation due to the primary mirrors and verified that also (a)-(d) can be accepted.

Regarding the mirror reflectivity, in the (c) and (d) we report, respectively, its average value and its nonuniformity for each segment. As for the MST segments, also for all the ASTRI COR2 and COR 3 segments, these parameters are compliant with the lower limit of $85 \%$ and the upper limit of $8 \%$, respectively.

In Figs. 16 and 17, the previous results are summarized in histograms, which report the distribution of each parameter around its average value.
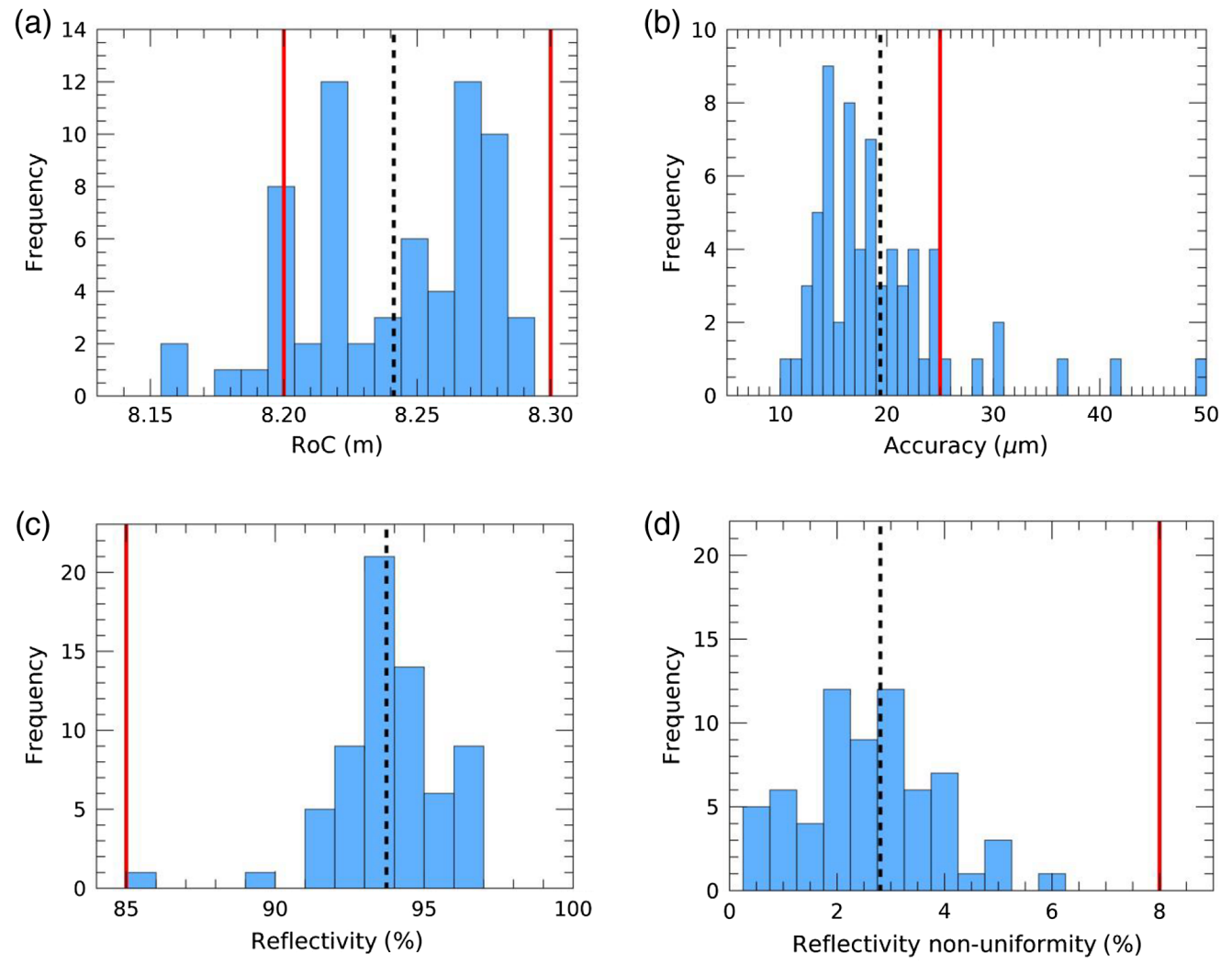

Fig. 17 Distribution of the main parameters for the COR3 segments: (a) radius of curvature, (b) residual shape error, (c) average reflectivity, and (d) reflectivity nonuniformity. For each panel, the red solid and black dashed lines represent, respectively, the applicable requirement value and the average value. 


\section{Acknowledgments}

Part of the results reported in this paper were anticipated in 2019 at the SPIE Conference 11119 "Optics for EUV, X-Ray, and Gamma-Ray Astronomy IX" and were reported in the relevant SPIE Proceedings (La Palombara et al. 2019, SPIE Proc. 111191U). This work was supported by the Italian Ministry of University and Research with funds specifically assigned to the Italian National Institute of Astrophysics for the Cherenkov Telescope Array (CTA) and the Italian Ministry of Economic Development within the "Astronomia Industriale" program. We acknowledged support from Brazilian Funding Agency FAPESP (Grant No. 2013/10559-5) and the South African Department of Science and Technology through Funding Agreement No. 0227/2014 for the South African Gamma-Ray Astronomy Program. This work was conducted in the context of the CTA SST and MST Working Groups. We gratefully acknowledged financial support from the agencies and organizations listed here. ${ }^{58}$ We would like to thank Dr. G. Tagliaferri, who coordinates the INAF participation to the MST Consortium, for his support to this activity. We also would like to thank Dr. G. Grisoni (M. L.) for providing us with useful comments and suggestions. This work has gone through internal review by the ASTRI Collaboration and by the CTA Consortium.

\section{References}

1. M. de Naurois and D. Mazin, "Ground-based detectors in very-high-energy gamma-ray astronomy," C. R. Phys. 16(6), 610-627 (2015).

2. T. C. Weekes et al, "Observation of TeV gamma rays from the crab nebula using the atmospheric Cerenkov imaging technique," Astrophys. J. 342, 379-395 (1989).

3. F. Aharonian et al., "Observations of the Crab Nebula with HESS," Astron. Astrophys. 457(3), 899-915 (2006).

4. J. Aleksic et al., "Performance of the MAGIC stereo system obtained with Crab Nebula data," Astropart. Phys. 35(7), 435-448 (2012).

5. N. Park and VERITAS Collaboration, "Performance of the VERITAS experiment," in 34th Int. Cosmic Ray Conf., ICRC, Vol. 34, pp. 771 (2015).

6. S. Wakely and D. Horan, "Welcome to TeVCat!," http://tevcat.uchicago.edu/ (2022).

7. H. Abdalla et al., "The H.E.S.S. galactic plane survey," Astron. Astrophys. 612, A1 (2018).

8. V. Vassiliev, S. Fegan, and P. Brousseau, "Wide field aplanatic two-mirror telescopes for ground-based $\gamma$-ray astronomy," Astropart. Phys. 28, 10-27 (2007).

9. E. Giro et al., "First optical validation of a Schwarzschild-Couder telescope: the ASTRI SST-2M Cherenkov telescope," Astron. Astrophys. 608, A86 (2017).

10. CTAO gGmbH, "CTA prototype telescope, ASTRI, achieves first light," https://www.ctaobservatory.org/cta-prototype-telescope-astri-achieves-first-light/ (2017).

11. S. Lombardi et al., "First detection of the Crab Nebula at TeV energies with a Cherenkov telescope in dual-mirror Schwarzschild-Couder configuration: the ASTRI-Horn telescope," Astron. Astrophys. 634, A22 (2020).

12. G. Pareschi, "The ASTRI SST-2M prototype and mini-array for the Cherenkov Telescope Array (CTA)," Proc. SPIE 9906, 99065 T (2016).

13. S. Scuderi, "From the Etna volcano to the Chilean Andes: ASTRI end-to-end telescopes for the Cherenkov Telescope Array," Proc. SPIE 10700, $107005 Z$ (2018).

14. L. A. Antonelli, "The ASTRI mini-array at Teide observatory," in Proc. ICRC, Vol. 37 , p. 832 (2021)

15. INAF News, "ASTRI: a new pathfinder of the arrays of Cherenkov telescopes," http://www .inaf.it/en/inaf-news/astri-a-new-pathfinder-of-the-arrays-of-cherenkov-telescopes (2019).

16. B. S. Acharya et al., "Introducing the CTA concept," Astropart. Phys. 43, 3-18 (2013).

17. B. S. Acharya et al., Science with the Cherenkov Telescope Array, World Scientific Publishing Co. Pte. Ltd., Singapore (2019).

18. R. Zanin, "CTA: the World's largest ground-based gamma-ray observatory," in Proc. ICRC, Vol. 37, p. 1478 (2021).

19. CTAO gGmbh, “CTAO's expected “Alpha Configuration” performance," https://www.ctaobservatory.org/science/ctao-performance/. 
La Palombara et al.: Mirror production for the Cherenkov telescopes of the ASTRI mini-array...

20. J. Rousselle, "Toward the construction of a medium size prototype Schwarzschild-Couder telescope for CTA," Proc. SPIE 9603, 960305 (2015).

21. C. Adams et al., "Alignment of the optical system of the 9.7-m prototype SchwarzchildCoulder Telescope," Proc. SPIE 11445, 114456A (2020).

22. G. Pareschi et al., "The dual-mirror Small Size Telescope for the Cherenkov Telescope Array," in Proc. ICRC, Vol. 33, p. 0466 (2013).

23. R. White et al., "The small-sized telescopes for the southern site of the Cherenkov Telescope Array," in Proc. ICRC, Vol. 37, p. 638 (2021)

24. J. M. Davies and E. S. Cotton, "Design of the quartermaster solar furnace," Solar Energy 1(2), 16-22 (1957)

25. G. Pareschi et al., "Glass mirrors by cold slumping to cover $100 \mathrm{~m}^{2}$ of the MAGIC II Cherenkov telescope reflecting surface," Proc. SPIE 7018, 70180W (2008).

26. R. Canestrari and G. Sironi, "An overview on mirrors for Cherenkov telescopes manufactured by glass cold-shaping technology," Proc. SPIE 9603, 960302 (2015).

27. R. Canestrari et al., "The glass cold-shaping technology for the mirrors of the Cherenkov Telescope Array," Proc. SPIE 9151, 91512V (2014).

28. R. Canestrari et al., "The ASTRI SST-2M prototype for the next generation of Cherenkov telescopes: structure and mirrors," Proc. SPIE 8861, 886102 (2013).

29. M. Garczarczyk, "Medium size telescopes for the Cherenkov Telescope Array," Proc. SPIE 10700, 1070023 (2018).

30. A. Förster et al., "Mirror development for CTA," Proc. SPIE 7437, 743712 (2009).

31. A. Förster et al., "Mirror development for the Cherenkov Telescope Array," in Proc. ICRC, Vol. 33, p. 0747 (2013)

32. G. Pareschi et al., "Status of the technologies for the production of the Cherenkov Telescope Array (CTA) mirrors," Proc. SPIE 8861, 886103 (2013).

33. M. Actis et al., "Design concepts for the Cherenkov Telescope Array CTA: an advanced facility for ground-based high-energy gamma-ray astronomy," Exp. Astron. 32, 193-316 (2011).

34. J. L. Dournaux et al., "Aspherical mirrors for the Gamma-ray Cherenkov Telescope, a Schwarschild-Couder prototype proposed for the future Cherenkov Telescope Array," Proc. SPIE 9912, 991240 (2016).

35. K. Hari Krishnan, et al., "An overall aspect of electroless Ni-P depositions: a review," Metallurg. Mater. Trans. 37(6), 1917-1926 (2006)

36. R. Graue and G. Valsecchi, "Superfinish technology for enhanced grazing incidence reflectivity in X-ray telescopes," Proc. SPIE 2805, 293-300 (1996).

37. Y. Namba et al., "Ultra-precision polishing of electroless nickel molding dies for shorter wavelength applications," CIRP Ann. 57(1), 337-340 (2008)

38. D. Vernani et al., "Development of cold-slumping glass mirrors for imaging Cherenkov telescopes," Proc. SPIE 7018, 70180V (2008).

39. R. Canestrari et al., "Cold-shaping of thin glass foils as a method for mirror processing: from basic concepts to mass production of mirrors," Opt. Eng. 52, 051204 (2013).

40. K. Tayabaly et al., "Roughness tolerances for Cherenkov telescope mirrors," Proc. SPIE 9603, 960307 (2015).

41. J. M Bennett and L. Mattsson, Introduction to Surface Roughness and Scattering, Optical Society of America (1989)

42. R. Canestrari et al., "Techniques for the manufacturing of stiff and lightweight optical mirror panels based on slumping of glass sheets: concepts and results," Proc. SPIE 7437, 743711 (2009).

43. C.B. Adams et al, "Verification of the optical system of the 9.7-m prototype SchwarzschildCouder Telescope," Proc. SPIE 11488, 1148805 (2020).

44. C.B. Adams et al, "Detection of the Crab Nebula with the $9.7 \mathrm{~m}$ prototype SchwarzschildCouder telescope," Astropart. Phys. 128, 102562 (2021).

45. M. Dyrda et al., "Open-structure composite mirrors for the Cherenkov Telescope Array," in Proc. ICRC, Vol. 33, p. 0281 (2013)

46. P. Brun et al., "Composite mirror facets for ground-based gamma ray astronomy," Nucl. Instrum. Methods Phys. Res. Sect. A 714, 58 (2013). 
La Palombara et al.: Mirror production for the Cherenkov telescopes of the ASTRI mini-array...

47. M. Hayashida et al., "The optical system for the large size telescope of the Cherenkov Telescope Array," in Proc. ICRC, Vol. 34, p. 927 (2015)

48. T. Inada et al., "Design and production of segment mirrors for the large-sized telescopes of the Cherenkov Telescope Array," Proc. SPIE 11451, 114510G (2020).

49. M. Will et al., "Novel back-coated glass mirrors for the MAGIC telescopes," in Proc. ICRC, Vol. 36, p. 823 (2019).

50. G. Pareschi et al., "Aluminum-based segmented mirrors for gamma-ray Cherenkov telescopes: status and perspectives," Proc. SPIE 11822, 1182203 (2021).

51. R. Canestrari et al., "The ASTRI SST-2M prototype for the Cherenkov Telescope Array: manufacturing of the structure and the mirrors," Proc. SPIE 9145, 91450M (2014).

52. M. Garczarczyk, "Reflectivity of the MAGIC-II reflector," MAGIC Document 111003 (2011).

53. M. Garczarczyk, "Report from the Chile mirror test campaign," CTAO MST-STR-RD36144000-00011-2 Issue 1 (2021).

54. K. Tayabaly et al., "Point spread function computation in normal incidence for rough optical surfaces," Proc. SPIE 9911, 99111X (2016).

55. G. Sironi et al., "The Cherenkov optics qualification facilities at INAF-OAB laboratories," Nucl. Instrum. Methods Phys. Res. A 952, 162163 (2020).

56. A. Schulz et al., "Methods for the characterization of mirror facets for Imaging Atmospheric Cherenkov Telescopes," in Proc. ICRC, Vol. 32, p. 0580 (2011).

57. J. Dick et al., "Recent developments for the testing of Cherenkov Telescope Array mirrors and actuators in Tübingen," in Proc. ICRC, Vol. 34, p. 1040 (2015)

58. CTAO gGmbh, "Acknowledgments," http://www.cta-observatory.org/consortium acknowledgments.

Nicola La Palombara received his MS degree in physics from the University of Milano in 1992. He is a senior staff technologist at INAF-IASF Milano. He is the first or co-author of more than 60 refereed scientific and technical papers and more than 50 contributions to conferences. $\mathrm{He}$ studies galactic high-energy sources and currently is in charge of the product assurance activities for the ASTRI mini-array and the CTA SST projects.

Biographies of the other authors are not available. 